\title{
Eficácia da Solução de Cloreto de Sódio a 7,5\% com e sem Dextran 70 a 6\% na Preservação da Função Renal de Cães Hipovolêmicos Submetidos à Isquemia-Reperfusão *
}

\author{
Efficacy of 7.5\% Hypertonic Sodium Chloride, with and without \\ $6 \%$ Dextran 70, in Renal Function Preservation of Hypovolemic \\ Dogs Submitted to Ischemia-Reperfusion
}

Geraldo Rolim Rodrigues Júnior, TSA ${ }^{1}$, José Luiz Gomes do Amaral, TSA ${ }^{2}$,

Yara Marcondes Machado Castiglia, TSA ${ }^{3}$, Mariangela Esther Alencar Marques ${ }^{4}$

\begin{abstract}
RESUMO
Rodrigues Júnior GR, Amaral JLG, Castiglia YMM, Marques MEA - Eficácia da Solução de Cloreto de Sódio a 7,5\% com e sem Dextran 70 a $6 \%$ na Preservação da Função Renal de Cães Hipovolêmicos Submetidos à Isquemia-Reperfusão
\end{abstract}

Justificativa e Objetivos - As soluções hipertônicas de cloreto de sódio, associadas ou não a colóides hiperoncóticos, podem ser eficazes em proteger o rim em situações de hipovolemia. 0 objetivo deste estudo foi verificar, em cães, o real benefício dessas soluções sobre a função renal, em vigência de hipovolemia e isquemia do órgão.

Método - Em 24 cães, anestesiados com pentobarbital sódico, submetidos à nefrectomia direita e à expansão volêmica com solução de Ringer $\left(1 \mathrm{ml} \cdot \mathrm{kg}^{-1} \cdot \mathrm{min}^{-1}\right)$, foram observadas possíveis alterações renais morfo-funcionais após hemorragia de $20 \mathrm{ml} . \mathrm{kg}^{-1}$ e trinta minutos de total isquemia renal esquerda, com posterior reperfusão, além da repercussão renal da administração de soluções de cloreto de sódio 7,5\% (SH) e esta em dextran 70 a $6 \%$ (SHD). Atributos estudados: FC, PAM, pressão de veia cava inferior, fluxo sangüíneo renal, resistência vascular renal, hematócrito, $\mathrm{Na}^{+}, \mathrm{K}^{+}$, osmolaridade plasmática, $\mathrm{PaO}_{2}, \mathrm{PaCO}_{2}$ e pH, depuração (para-aminohipurato de sódio - $\mathrm{PAH}_{-1}$, creatinina, osmolar, água livre, $\mathrm{Na}^{+}, \mathrm{K}^{+}$), fração de filtração, volume e osmolaridade urinários, excreções urinárias e fracionárias de $\mathrm{Na}^{+}$e $\mathrm{K}^{+}$e exame histopatológico do rim. Os atributos foram estudados em três grupos (G1, G2 e G3) e em cinco momentos.

Resultados - Houve elevação estatisticamente significativa da pressão arterial média em G2 e G3, da resistência vascular renal em G1, do fluxo sangüíneo renal e da depuração de PAH em

* Recebido do (Received from) Departamento de Anestesiologia da Faculdade de Medicina de Botucatu - UNESP

1. Professor Assistente Doutor do Departamento de Anestesiologia da FMB - UNESP

2. Professor Titular da Disciplina de Anestesiologia, Dor e Terapia Intensiva Cirúrgica da EPM/UNIFESP

3. Professora Titular do Departamento de Anestesiologia da FMB UNESP

4. Professora Assistente Doutora do Departamento de Patologia da FMB UNESP

Apresentado (Submitted) em 29 de maio de 2001

Aceito (Accepted) para publicação em 08 de agosto de 2001

Correspondência para mail:

Dr. Geraldo Rolim Rodrigues Júnior

Dept ${ }^{\circ}$ de Anestesiologia da FMB - UNESP

Distrito de Rubião Junior

18618-970 Botucatu, SP

E-mail: anestesi@fmb.unesp.br

(c) Sociedade Brasileira de Anestesiologia, 2002
G3, da excreção fracionária de $\mathrm{Na}^{+}$em G2 e G3, das depurações de creatinina, osmolar, de água livre e de $\mathrm{Na}^{+}$e $\mathrm{K}^{+}$, da excreção urinária de $\mathrm{Na}^{+}$e $\mathrm{K}^{+}$e do volume urinário em $\mathrm{G} 3$.

Conclusões - $A$ SHD administrada 15 minutos após hemorragia moderada e 30 minutos antes de insulto isquêmico de 30 minutos foi eficiente em proteger o rim de cães das repercussões da isquemia-reperfusão. Não foi constatada alteração histopatológica renal à microscopia óptica.

UNITERMOS - ANIMAL: cão; VOLEMIA: expansão, colóide, solução de $\mathrm{NaCl}$ a $7,5 \%$

\section{SUMMARY}

Rodrigues Júnior GR, Amaral JLG, Castiglia YMM, Marques MEA - Efficacy of $7.5 \%$ Hypertonic Sodium Chloride, with and without $6 \%$ Dextran 70, in Renal Function Preservation of Hypovolemic Dogs Submitted to Ischemia-Reperfusion

Background and Objectives - Sodium chloride hypertonic solutions, with or without hyperoncotic colloids, may be effective in protecting kidney against hypovolemia. This experiment aimed at evaluating, in dogs, the real benefit of these solutions on renal function in the presence of hypovolemia and ischemia.

Methods - Experiments were performed in 24 mixed-breed dogs anesthetized with sodium pentobarbital, submitted to right nephrectomy and volume expansion with Ringer's solution (1 $\left.\mathrm{ml} \cdot \mathrm{kg}^{-1} \cdot \mathrm{min}^{-1}\right)$. Renal morphological and physiological changes were studied after $20 \mathrm{ml} . \mathrm{kg}^{-1}$ hemorrhage and $30 \mathrm{~min}$ of total left renal ischemia followed by reperfusion, in addition to renal repercussion of $7.5 \% \mathrm{NaCl}$ (HS) and $7.5 \% \mathrm{NaCl}$ in $6 \%$ dextran 70 (HSD). The following parameters were studied: HR, MBP, inferior vena cava pressure, renal blood flow, renal vascular resistance, hematocrit, $\mathrm{Na}^{+}, \mathrm{K}^{+}$, plasma osmolarity, $\mathrm{PaO}_{2}, \mathrm{PaCO}_{2}$ and $\mathrm{pH}$; clearance (para-aminohipuric acid - $\mathrm{PAH}_{-1}$, creatinine, free water, osmolar, $\mathrm{Na}^{+}$and $\left.\mathrm{K}^{+}\right)$, filtration fraction, urine output and osmolarity, sodium and potassium urine and fractionated excretions; rectal temperature; and kidney histopathology. Atributes where studied in five moments in three groups (G1, G2 and G3).

Results - There has been a statistically significant increase in mean blood pressure in $G 2$ and G3. RVR was higher in G1; RBF and PAH clearance were higher in $\mathrm{G} 3 ; \mathrm{Na}^{+}$fractionated excretion was increased in G2 and G3; creatinine, free water, osmolar, $\mathrm{Na}^{+}$and $\mathrm{K}^{+}$clearances, diuresis, and urinary excretions of $\mathrm{Na}^{+}$and $\mathrm{K}^{+}$were higher in $\mathrm{G} 3$.

Conclusions - HSD infusion 15 min after mild hemorrhage and 30 min before ischemia was effective in protecting kidney of dogs from ischemia-reperfusion repercussions. There have been no histopathologic changes under optical microscopy.

KEY WORDS - ANIMAL: dog; VOLEMIA: expansion, colloid, $7.5 \% \mathrm{NaCl}$ solution 


\section{INTRODUÇÃO}

As soluções hipertônicas de cloreto de sódio, em baixos volumes, restauram rapidamente o volume intravascular porque redistribuem a água, a partir do compartimento intracelular. Este aumento do volume sangüíneo é transitório, entretanto ocorre mais rapidamente que durante infusão, no mesmo período de tempo, de fluidos isosmóticos. Além disso, essas soluções podem melhorar o desempenho de vários órgãos, inclusive do coração, seja por efeito na contratilidade, por diminuição da pós-carga ou até por determinar varredura de radicais livres do oxigênio no miocárdio. Esse último efeito é produzido pelo dextran 70 , quando associado à solução de cloreto de sódio a $7,5 \%{ }^{1}$. Apesar de melhorar a perfusão de vários órgãos, inclusive do rim, durante a reanimação do choque hemorrágico, essas soluções tinham seu emprego limitado devido à brevidade de seus efeitos benéficos.

Atualmente, a utilização de baixos volumes de soluções hipertônicas de cloreto de sódio a $7,5 \%$, para o tratamento dos estados de hipovolemia, hipotensão e choque, difundiu-se com a associação de dextran 70 a $6 \%$. Tal formulação tornou mais duradouro o efeito dessas soluções e potencializou os efeitos fisiológicos benéficos de ambos os componentes ${ }^{1}$. Vários estudos em animais experimentais demonstram a eficiência dessas soluções em atenuar e até mesmo reverter uma série de anormalidades provocadas pelo choque hemorrágico e hipovolemia. Nessas situações, uma das principais preocupações é a preservação dos rins, cuja função, de importância fundamental para a homeostase do indivíduo, pode ser seriamente afetada, chegando a diversos graus de insuficiência, o que compromete a recuperação do doente.

A interrupção temporária do fluxo sangüíneo renal ocasiona alterações anatômicas e fisiológicas que terão tanto maior gravidade quanto maior for o tempo de isquemia. Trinta minutos de isquemia, associados à hipovolemia prévia, produzirão lesões renais graves, se não houver abrandamento ou prevenção, por medidas de proteção desse órgão.

As soluções hipertônicas de cloreto de sódio, associadas ou não a colóides hiperoncóticos, podem ser eficazes em proteger o rim nessas situações previsíveis. Vários autores sugerem que a chamada reanimação hipertônica do choque hemorrágico certamente reverte a disfunção renal presente nesses estados ${ }^{2}$. Outros pesquisadores afirmam que a solução de cloreto de sódio a $7,5 \%$, associada ao dextran 70 , possui a capacidade de reverter alguns dos efeitos celulares deletérios causados pela isquemia ou pela isquemia-reperfusão, principalmente aqueles devidos ao aumento da perfusão capilar ${ }^{3}$.

Neste experimento, serão analisados os reais benefícios da solução hipertônica de cloreto de sódio, na concentração de $7,5 \%(\mathrm{SH})$, isoladamente e associada ao dextran 70 a $6 \%$ (SHD), sobre a simulação, no cão, de função renal alterada por hipovolemia associada à isquemia de 30 minutos.

\section{MÉTODO}

Após aprovação pelos Comitês de Ética em Pesquisa Experimental da Faculdade de Medicina de Botucatu (UNESP) e da EPM/UNIFESP, foram utilizados 24 cães adultos sem raça ou idade definidas, machos e fêmeas, com pesos variando de 7 a $33 \mathrm{~kg}$. Os animais foram anestesiados com pentobarbital sódico (PS), por via venosa, $30 \mathrm{mg} \cdot \mathrm{kg}^{-1}$ para indução e $5 \mathrm{mg} \cdot \mathrm{kg}^{-1}$ para manutenção (aos 105 minutos após a dose inicial).

Os animais foram distribuídos aleatoriamente em 3 grupos experimentais, formados por 8 cães cada.

Grupo 1 (G1) - Os cães foram submetidos à lombotomia direita para retirada do rim direito e lombotomia esquerda para canulização ureteral e pinçamento da artéria renal esquerda por 30 minutos. Todos os animais foram submetidos à hemorragia aproximada de $29 \%$ a $30 \%$ de seu volume sangüíneo, suficiente para causar uma redução do volume circulante que correspondesse ao estado de hipovolemia, objetivo do estudo, e não ao choque.

Grupo 2 (G2) - Mesmos procedimentos do grupo 1, seguidos da administração por via venosa, 15 minutos após a hemorragia programada, de $\mathrm{SH}$, na dose de $4 \mathrm{ml} . \mathrm{kg}^{-1} \mathrm{em} 3$ minutos.

Grupo 3 (G3) - Mesmos procedimentos do grupo 1, seguidos da administração venosa de SHD, na mesma dose e velocidade utilizadas no grupo 2 .

Após jejum de 14 horas, os animais foram anestesiados com pentobarbital sódico e colocados em decúbito dorsal sobre a goteira de Claude Bernard. Realizou-se a seguir:

1. Intubação orotraqueal e instalação de ventilação controlada com ar, empregando-se aparelho de anestesia K. Takaoka, Mod 850-10. O volume corrente foi de 15 $\mathrm{ml} . \mathrm{kg}^{-1}$ e a freqüência respiratória, de 15 movimentos por minuto;

2. Dissecção e cateterismo da veia femoral esquerda para medida de pressão da veia cava inferior, através de manômetro de água, administração das doses complementares de pentobarbital sódico e de cloreto de alcurônio $\left(0,2 \mathrm{mg} \cdot \mathrm{kg}^{-1}\right)$, inicialmente, e $0,006 \mathrm{mg} \cdot \mathrm{kg}^{-1}$ para doses complementares, coleta de sangue venoso, dosagem bioquímica e infusão contínua de solução de Ringer ( $\left.1 \mathrm{ml} \cdot \mathrm{kg}^{-1} \cdot \mathrm{min}^{-1}\right)$; dissecção e cateterismo da artéria femoral direita para medida de pressão arterial média, através de manômetro de mercúrio, e da femoral esquerda para retirada de volume sangüíneo correspondente a $20 \mathrm{ml} . \mathrm{kg}^{-1}$;

3. Dissecção e cateterismo da veia femoral direita para administração, 30 minutos após o início da infusão contínua de solução de Ringer, da dose prime de $\mathrm{PAH}$ $\left(4 \mathrm{mg} \cdot \mathrm{kg}^{-1}\right)$ e de creatinina ( $\left.30 \mathrm{mg} \cdot \mathrm{kg}^{-1}\right)$ em solução de Ringer. A seguir, foi instalada e mantida até o término da experiência infusão contínua da solução de $\mathrm{PAH}$ $(0,06 \mathrm{~g} \%)$ e de creatinina $(0,15 \mathrm{~g} \%)$ em solução de Ringer, sendo administrados, por minuto, 0,6 mg de creati- 
nina e $0,24 \mathrm{mg}$ de $\mathrm{PAH}$ por quilograma de peso do animal $\left(0,4 \mathrm{ml} \cdot \mathrm{kg}^{-1} \cdot \mathrm{min}^{-1}\right)$;

4. Realização de lombotomia à direita para a retirada do rim direito e à esquerda para a cateterização do ureter e dissecção do pedículo renal esquerdo; exposição da artéria renal esquerda, passagem de pinça não traumática, a qual permaneceria aberta até o momento do pinçamento;

5. Cateterização do ureter com sonda de polivinil de números 6 ou 8, dependendo do animal. A urina, sempre que era produzida, fluía continuamente pela sonda durante o experimento;

6. Colocação de termômetro de álcool no reto para determinação da temperatura;

7. Retirada de volume sangüíneo pela artéria femoral esquerda, 30 minutos após a injeção da solução prime;

8. Injeção de cloreto de sódio a $0,9 \%$ no G1, a $7,5 \%$ no G2 e a $7,5 \%$ em dextran 70 a $6 \%$ no G3, 15 minutos após a hemorragia e 45 minutos após a injeção da solução prime;

9. Pinçamento da artéria renal esquerda em todos os grupos, com despinçamento 30 minutos após;

10. Sacrifício do animal com formol a $20 \%$ IV e retirada do rim esquerdo para estudo histopatológico.

Os atributos foram classificados em dois grupos.

a) Para controle da homogeneidade: antropométricos peso do animal $(\mathrm{kg})$, sexo e comprimento $\left(\mathrm{m}^{-1}\right)$; freqüência respiratória $\left(\mathrm{mov} \cdot \mathrm{min}^{-1}\right)$, volume corrente $\left(\mathrm{ml} . \mathrm{kg}^{-1}\right)$, hematócrito $(\mathrm{Ht})(\%)$, temperatura retal $\left({ }^{\circ} \mathrm{C}\right)$;

b) Para atender às finalidades da pesquisa: hemodinâmicos: pressão arterial média (PAM); pressão da veia cava inferior $(\mathrm{PCl})$; freqüência cardíaca $(\mathrm{FC})$; fluxo plasmático renal (medido pela depuração de $\mathrm{PAH}-\mathrm{D}_{\mathrm{PAH}}=\mathrm{U}_{\mathrm{PAH}} \times \mathrm{V} / \mathrm{P}_{\mathrm{PAH}}$; na qual $\mathrm{V}=$ débito urinário, $\mathrm{U}$ e $\mathrm{P}=$ concentração de PAH na urina e plasma, respectivamente); fluxo sangüíneo renal (FSR = $\left.\mathrm{D}_{\mathrm{PAH}} / 1-\mathrm{Ht}\right)$; resistência vascular renal $(\mathrm{RVR}=$ PAM/FSR). De função renal: ritmo de filtração glomerular (RFG) (medido pela depuração da creatinina $\left.-D_{c r}=U_{c r} \times V / P_{c r}\right)$; débito urinário $(V)$; fração de filtração ( $\left.F F=D_{c r} / D_{\mathrm{PAH}}\right)$; osmolaridade plasmática $\left(\mathrm{P}_{\text {osm }}\right)$; osmolaridade urinária $\left(\mathrm{U}_{\mathrm{osm}}\right)$; depuração osmolar $\left(\mathrm{D}_{\text {osm }}=\right.$ Uosm $\left.\times \mathrm{V} / \mathrm{P}_{\text {osm }}\right)$; depuração de sódio e potássio $\left(D_{\mathrm{Na} \text { ou K }}=U_{\mathrm{Na} \text { ou K }} \times \mathrm{V} / \mathrm{P}_{\mathrm{Na} \text { ou K }}\right)$; depuração de água livre $\left(\mathrm{D}_{\mathrm{H}_{2} \mathrm{O}}=\mathrm{V}-\mathrm{C}_{\mathrm{osm}}\right)$; excreção urinária de sódio $\left(\mathrm{U}^{+}{ }_{\mathrm{nu}} \times \mathrm{V}=\mathrm{U}_{\mathrm{Na}} \times \mathrm{V}\right)$; excreção fracionária de sódio $\left(E F_{\mathrm{Na}}=D_{\mathrm{Na}} / D_{\mathrm{cr}} \times 100\right)$; excreção urinária de potássio $\left(\mathrm{U}_{\mathrm{K}} \mathrm{V}=\mathrm{K}_{\mathrm{u}}^{+} \times \mathrm{V}\right)$; excreção fracionária de potássio (EF $\left.=D_{K} / D_{c r} \times 100\right)$. Estudo histopatológico do rim esquerdo.

Esses parâmetros foram obtidos nos momentos que se seguem e seus resultados comparados nos três grupos estudados. Não fez parte dos objetivos deste experimento medirvariáveis hemodinâmicas logo após hemorragia, mas, sim, ve- rificar eficiência de algumas soluções. Para determinar este impacto, estudou-se o grupo controle G1.

M1 e M2 - obtidos 15 e 60 minutos após injeção do prime de $\mathrm{PAH}$ e creatinina;

M3 e M4 - obtidos 105 e 120 minutos após injeção do prime de PAH e creatinina (imediatamente e 15 minutos após despinçamento, respectivamente); e M5 - obtido 135 minutos após injeção do prime de PAH e creatinina e 30 minutos após despinçamento.

Nos grupos G2 e G3, foram administradas, respectivamente, SH e SHD na dose de $4 \mathrm{ml} . \mathrm{kg}^{-1}$ de peso do animal, para reposição da hemorragia, 15 minutos após a retirada do volume sangüíneo. No G1, foi administrada a mesma dose de cloreto de sódio a $0,9 \%$.

Quadro I - Drogas e Soluções Administradas Durante a Seqüência Experimental

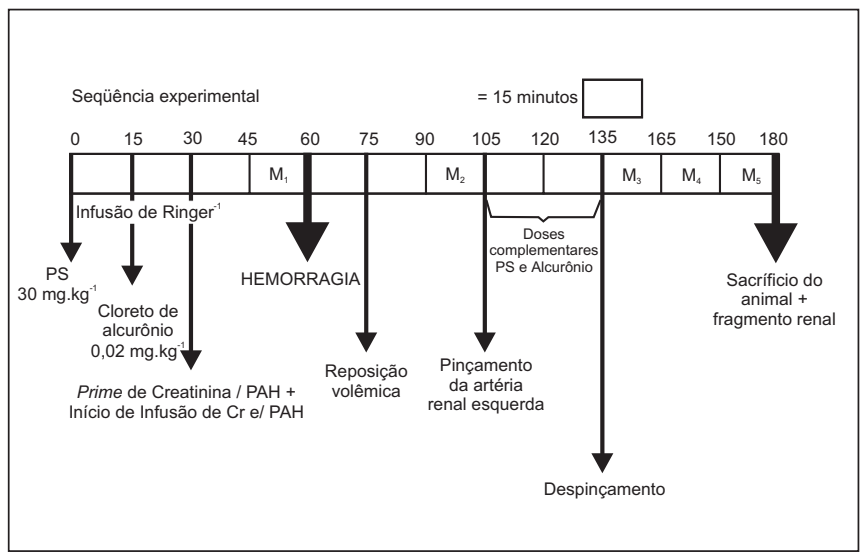

O fragmento selecionado para a histopatologia foi colocado em solução de formol a $5 \%$. Após ser fixado por um período superior a 48 horas, foi lavado e incluído em parafina, para depois ser cortado e corado pela hematoxilina-eosina e ácido periódico de Schiff (PAS). As lâminas foram rotuladas e analisadas sem o prévio conhecimento do grupo experimental a que pertenciam.

Foi utilizada a análise de perfil de Morrison para cada variável e fixou-se p em 0,05 ou $5 \%(\alpha<$ ou $=0,05)$ o nível de rejeição da hipótese de nulidade, assinalando-se com um asterisco os valores significantes. No caso de $0,05<p<0,10$, foi referida tendência à significância. Os contrastes entre médias de grupos foram verificados calculando-se a diferença mínima significativa, para $\alpha=0,05$, pelo método de Tukey. Parâmetros antropométricos foram avaliados pela análise de variância (ANOVA).

\section{RESULTADOS}

Os resultados mais significativos estão representados nas tabelas I, II e III e figuras 1, 2, 3 e 4.

Houve homogeneidade (ANOVA) nos parâmetros peso, comprimento e sexo entre os cães dos três grupos experimentais. Os atributos $\mathrm{U}_{\mathrm{osm}}, \mathrm{C}_{\mathrm{H}_{2} \mathrm{O}}, \mathrm{EF}_{\mathrm{Na}}, \mathrm{EF}_{\mathrm{K}}, \mathrm{PaO}_{2}, \mathrm{PaCO}_{2}$, $\mathrm{pH}, \mathrm{PCl}, \mathrm{P}_{\mathrm{osm}}, \mathrm{FF}, \mathrm{V}, \mathrm{C}_{\mathrm{osm}}, \mathrm{C}_{\mathrm{Na}}, \mathrm{U}_{\mathrm{Na}} \mathrm{V}, \mathrm{C}_{\mathrm{K}} \mathrm{e} \mathrm{U}_{\mathrm{K}} \mathrm{V}$ não apresenta- 
Tabela I - Fluxo Sangüíneo Renal (FSR) e Resistência Vascular Renal (RVR). Média e Desvio Padrão em cada Momento nos 3 Grupos Experimentais

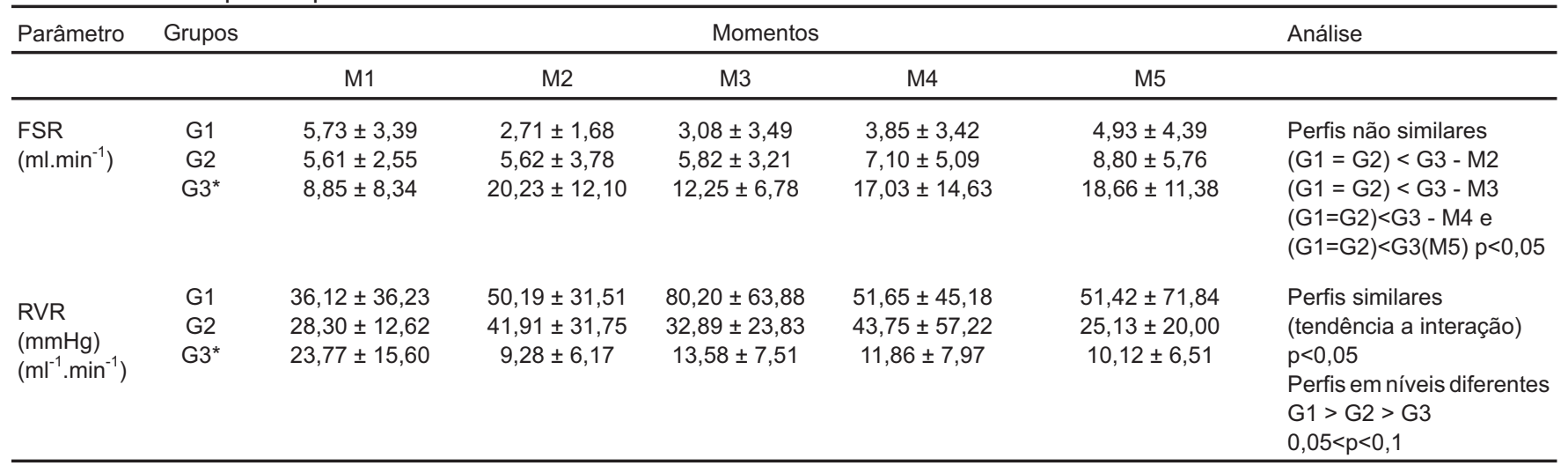

Comentário: Em relação a FSR, apenas G3* apresentou relevância estatística e valores superiores aos dos demais grupos no decorrer do experimento. A RVR foi decrescente, sendo maior no controle (G1) e menor em G3* (tendência à interação), que teve a menor resistência dentre os grupos

Tabela II - Atributos de Sódio $\left(\mathrm{Na}^{+}\right)$- Plasmático, Depuração e Excreção Urinária - e Depuração Osmolar. Média e Desvio Padrão dos Valores Observados em Cada Momento nos 3 Grupos Experimentais

\begin{tabular}{|c|c|c|c|c|c|c|c|}
\hline \multirow[t]{2}{*}{ Parâmetro } & \multirow[t]{2}{*}{ Grupos } & & \multicolumn{3}{|c|}{ Momentos } & \multicolumn{2}{|r|}{ Análise } \\
\hline & & M1 & M2 & M3 & M4 & M5 & \\
\hline $\begin{array}{l}\text { Sódio } \\
\text { plasmático } \\
\left(\mathrm{mEq} \cdot \mathrm{I}^{-1}\right)\end{array}$ & $\begin{array}{l}\mathrm{G} 1 \\
\mathrm{G} 2 \\
\mathrm{G} 3^{*}\end{array}$ & $\begin{array}{l}144,00 \pm 2,62 \\
146,25 \pm 3,28 \\
145,75 \pm 2,25\end{array}$ & $\begin{array}{l}144,75 \pm 2,60 \\
151,25 \pm 3,19 \\
150,25 \pm 4,83\end{array}$ & $\begin{array}{l}146,25 \pm 2,25 \\
149,25 \pm 4,53 \\
149,75 \pm 3,28\end{array}$ & $\begin{array}{l}145,75 \pm 2,71 \\
148,25 \pm 5,17 \\
150,25 \pm 4,06\end{array}$ & $\begin{array}{l}145,75 \pm 1,98 \\
149,50 \pm 4,75 \\
148,50 \pm 3,16\end{array}$ & $\begin{array}{l}\text { Perfis em níveis diferentes } \\
\mathrm{G} 1<(\mathrm{G} 2=\mathrm{G} 3) \\
\mathrm{M} 1<(\mathrm{M} 2=\mathrm{M} 3=\mathrm{M} 4=\mathrm{M} 5) \\
\mathrm{p}<0,05\end{array}$ \\
\hline
\end{tabular}

$\begin{array}{llllllc}\text { Depuração } & \text { G1 } & 687,37 \pm 90,59 & 764,87 \pm 320,44 & 542,75 \pm 128,53 & 494,12 \pm 96,60 & 443,12 \pm 112,78 \\ \text { osmolar } & \text { G2 } & 668,50 \pm 179,69 & 704,37 \pm 132,30 & 539,62 \pm 148,64 & 491,62 \pm 98,43 & 435,50 \pm 84,60 \\ \left(\mathrm{ml}^{\prime} \mathrm{min}^{-1}\right) & \text { G3 }^{*} & 708,75 \pm 135,99 & 590,25 \pm 206,24 & 500,75 \pm 142,76 & 487,25 \pm 175,20 & 424,50 \pm 175,49\end{array}$

Momentos elevam-se com o tempo.p<0,05

$(\mathrm{G} 1=\mathrm{G} 2)<\mathrm{G} 3-\mathrm{M} 2$ $(\mathrm{G} 1=\mathrm{G} 2)<\mathrm{G} 3-\mathrm{M} 3$ $(\mathrm{G} 1=\mathrm{G} 2)<\mathrm{G} 3-\mathrm{M} 4$ G1<G3 e G2 interm -M5 $p<0,05$

$\begin{array}{lllllll}\text { Depuração } & \text { G1 } & 0,14 \pm 9,73 & 0,14 \pm 9,45 & 0,20 \pm 0,14 & 0,25 \pm 0,16 & 0,28 \pm 0,17 \\ \text { de sódio } & \text { G2 } & 0,13 \pm 5,78 & 0,41 \pm 0,29 & 0,45 \pm 0,36 & 0,53 \pm 0,51 & 0,73 \pm 0,69 \\ \left(\mathrm{ml}^{\prime} \mathrm{min}^{-1}\right) & \mathrm{G}^{*} & 0,32 \pm 0,21 & 1,31 \pm 0,45 & 1,02 \pm 0,33 & 1,20 \pm 0,49 & 1,28 \pm 0,34\end{array}$

(G1=G2)<G3-M1 $\mathrm{G} 1=\mathrm{G} 2)<\mathrm{G} 3-\mathrm{M} 2$ $(\mathrm{G} 1=\mathrm{G} 2)<\mathrm{G} 3-\mathrm{M} 3$ $(\mathrm{G} 1=\mathrm{G} 2)<\mathrm{G} 3-\mathrm{M} 4$ G1<G3 e G2 interm - M5 $p<0,05$

\begin{tabular}{|c|c|c|c|c|c|c|c|}
\hline Excreção & G1 & $20,51 \pm 13,94$ & $19,75 \pm 13,68$ & $28,95 \pm 20,19$ & $37,01 \pm 23,49$ & $41,53 \pm 25,16$ & \\
\hline urinária & G2 & $19,61 \pm 8,17$ & $61,54 \pm 44,40$ & $66,92 \pm 55,40$ & $80,50 \pm 78,11$ & $110,55 \pm 107,00$ & $(\mathrm{G} 1=\mathrm{G} 2)<\mathrm{G} 3-\mathrm{M} 1 \mathrm{p}<0,05$ \\
\hline $\begin{array}{l}\text { de sódio } \\
\left(\mathrm{mEq} \cdot \mathrm{min}^{-1}\right)\end{array}$ & $\mathrm{G}^{*}$ & $46,62 \pm 31,90$ & $197,93 \pm 70,40$ & $153,25 \pm 50,03$ & $181,21 \pm 76,98$ & $189,69 \pm 52,66$ & $\begin{array}{l}\mathrm{G} 1<\mathrm{G} 2<\mathrm{G} 3-\mathrm{M} 2 \\
\mathrm{G} 1<\mathrm{G} 2<\mathrm{G} 3-\mathrm{M} 3 \\
\mathrm{G} 1<\mathrm{G} 2<\mathrm{G} 3-\mathrm{M} 4 \\
\mathrm{G} 1<\mathrm{G} 2<\mathrm{G} 3-\mathrm{M} 5 \mathrm{p}<0,05\end{array}$ \\
\hline
\end{tabular}

Comentário: Todas as variáveis têm envolvimento do $\mathrm{Na}^{+}$, uma vez que esse íon é o responsável direto pela pressão osmótica. As soluções administradas em G3 e G2 determinaram elevação no $\mathrm{Na}^{+}$plasmático nesses grupos em relação ao controle. Ambas as depurações e a excreção urinária de $\mathrm{Na}^{+}$tiveram aumento mais acentuadas em $\mathrm{G}^{*}$ no decorrer do experimento

ram alterações significativas nos grupos e momentos estudados.

A temperatura retal apresentou redução uniforme e contínua no decorrer do experimento em todos os grupos.
Mesmo seguindo-se todos os passos adequados para identificar qualquer lesão visível à microscopia óptica, não foi evidenciada qualquer lesão compatível com necrose tubular aguda. 
EFICÁCIA DA SOLUÇÃO DE CLORETO DE SÓDIO A 7,5\% COM E SEM DEXTRAN 70 A $6 \%$ NA PRESERVAÇÃO DA FUNÇÃO RENAL DE CÃES HIPOVOLÊMICOS SUBMETIDOS À ISQUEMIA- REPERFUSÃO

Tabela III - Atributos de Potássio $\left(\mathrm{K}^{+}\right)$, Depuração e Excreção Urinária. Média e Desvio Padrão em cada Momento nos 3 Grupos Experimentais

\begin{tabular}{|c|c|c|c|c|c|c|c|}
\hline \multirow[t]{2}{*}{ Parâmetro } & \multirow[t]{2}{*}{ Grupos } & & \multicolumn{3}{|c|}{ Momentos } & \multicolumn{2}{|r|}{ Análise } \\
\hline & & M1 & M2 & M3 & M4 & M5 & \\
\hline \multirow{2}{*}{$\begin{array}{l}\text { Depuração } \\
\text { de potássio } \\
\left(\mathrm{ml} \cdot \mathrm{min}^{-1}\right)\end{array}$} & G1 & $2,87 \pm 1,57$ & $3,17 \pm 2,08$ & $2,95 \pm 2,40$ & $3,87 \pm 2,96$ & $3,40 \pm 2,51$ & $(\mathrm{G} 1=\mathrm{G} 2)<\mathrm{G} 3-\mathrm{M} 2$ \\
\hline & $\mathrm{G}^{*}$ & $5,35 \pm 1,87$ & $9,30 \pm 2,06$ & $10,30 \pm 1,46$ & $13,89 \pm 4,28$ & $13,41 \pm 2,92$ & $\begin{array}{l}\mathrm{G} 1<\mathrm{G} 2<\mathrm{G} 3-\mathrm{M} 4 \\
\mathrm{G} 1<\mathrm{G} 3<\mathrm{G} 2-\mathrm{M} 5 \\
\text { Momentos aumentam } \\
\text { com o tempo= G2-G3 } \\
\mathrm{p}<0,05\end{array}$ \\
\hline \multirow{3}{*}{$\begin{array}{l}\text { Excreção } \\
\text { urinária de } \\
\text { potássio } \\
\left(\mathrm{mEq} \cdot \mathrm{min}^{-1}\right)\end{array}$} & G1 & $11,11 \pm 6,15$ & $11,77 \pm 8,24$ & $10,81 \pm 9,44$ & $14,06 \pm 11,73$ & $14,09 \pm 9,75$ & $\mathrm{G} 1<\mathrm{G} 3$ e $\mathrm{G} 2$ interm - M2 \\
\hline & G2 & $16,91 \pm 10,81$ & $21,71 \pm 16,68$ & $23,68 \pm 11,00$ & $32,49 \pm 19,09$ & $38,74 \pm 17,14$ & $\mathrm{G} 1<\mathrm{G} 2<\mathrm{G} 3-\mathrm{M} 3$ \\
\hline & G3* & $19,96 \pm 6,51$ & $32,48 \pm 6,41$ & $39,05 \pm 5,92$ & $53,75 \pm 18,50$ & $49,16 \pm 9,92$ & $\begin{array}{l}\mathrm{G} 1<\mathrm{G} 2<\mathrm{G} 3-\mathrm{M} 4 \\
\mathrm{G} 1<(\mathrm{G} 2=\mathrm{G} 3)-\mathrm{M} 5 \\
\mathrm{p}<0,05\end{array}$ \\
\hline
\end{tabular}

Comentário: Os grupos que receberam soluções hipertônicas (mais acentuada em G3*) apresentaram maior depuração e excreção urinária de $\mathrm{K}^{+}$ (acentuadamente em G3) em relação ao controle (G1) no decorrer do experimento. Todavia, o $\mathrm{K}^{+}$plasmático não apresentou alterações

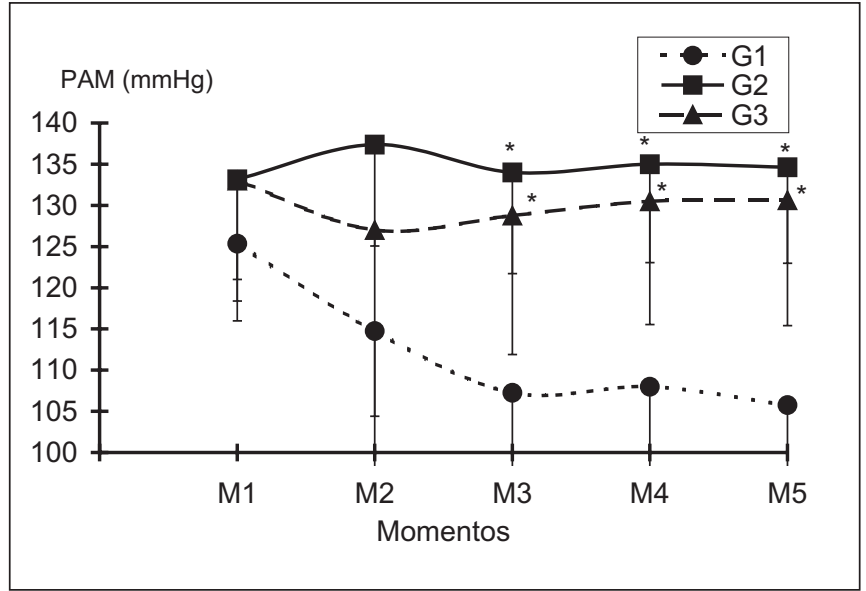

Figura 1 - Pressão Arterial Média (mmHg). Média e Desvio Padrão em Cada Momento nos 3 Grupos

Comentário: Após a hemorragia ( $30 \%$ da volemia) e posterior reposição (M1), a PAM teve diminuição significativa e progressiva em $\mathrm{H}^{*}$ * $(p<0,05)$ (controle) no decorrer do experimento, em relação aos demais grupos. Os grupo G3 * $(p<0,05)$, teve a maior PAM em M3, M4 e M5

\section{DISCUSSÃo}

A técnica anestésica utilizada é padrão em modelo canino por sua comprovada eficácia em atingir plano anestésico adequado em poucos minutos e por não apresentar efeitos na função renal.

O modelo de isquemia renal aguda em cães, já desenvolvido em pesquisa anterior, desta vez foi associado à hipovolemia provocada pela retirada aproximada de $29 \%$ do volume sangüíneo estimado do animal, método comprovadamente causador do estado de hipovolemia e utilizado por vários autores ${ }^{5}$.

Revista Brasileira de Anestesiologia

Vol. 52, № 1, Janeiro - Fevereiro, 2002

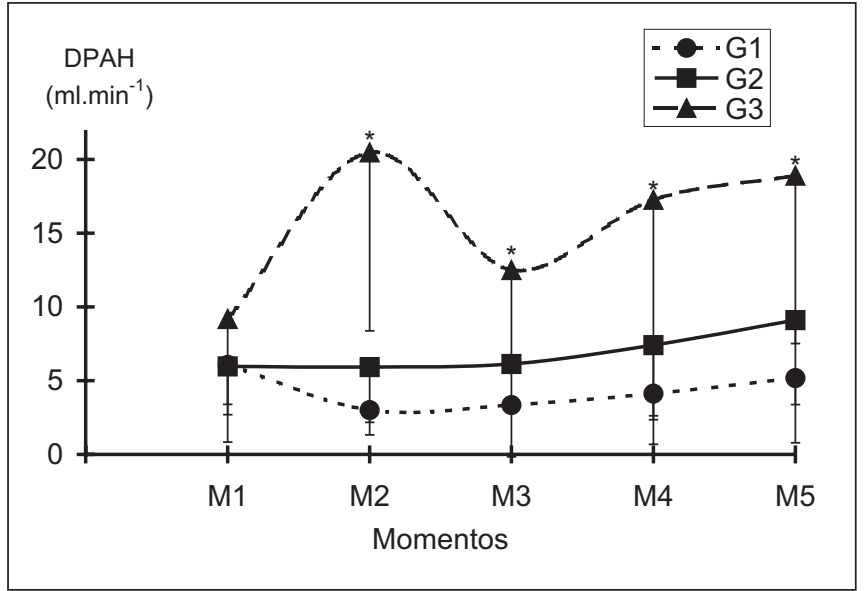

Figura 2 - Depuração PAH (Fluxo Plasmático Efetivo Renal) $\left(\mathrm{ml} \cdot \mathrm{min}^{-1}\right)$. Média e Desvio Padrão em Cada Momento nos 3 Grupos

Comentário: Após a hemorragia ( $30 \%$ da volemia) e posterior reposição (M1), a $\mathbf{D}_{\mathrm{PAH}}$ apresentou valores superiores aos dos demais grupos, apenas em G3* $(p<0,05)$, no decorrer do experimento

As conseqüências sobre a função renal, determinadas pela associação de hipovolemia e isquemia aguda, foram observadas no grupo 1 . Nos grupos 2 e 3 foi testada a ocorrência de uma proteção efetiva da função renal contra os efeitos deletérios dessa associação, utilizando-se as soluções $\mathrm{SH}$ e SHD.

A utilização de SH foi estabelecida em 1980 por Velasco e col. ${ }^{6}$, os quais observaram que uma solução de cloreto de sódio hipertônico a 7,5\%, cuja osmolaridade eqüivale a aproximadamente $2.400 \mathrm{mOsm} . \mathrm{I}^{-1}$, e na dose de $4 \mathrm{ml} . \mathrm{kg}^{-1}$ induz expansão máxima de plasma no tempo zero, de modo idêntico a uma solução de $883 \mathrm{mOsm} . \mathrm{I}^{-1}$ e em dose ${ }^{7}$ de $12 \mathrm{ml} \mathrm{kg}^{-1}$. Vários autores demonstraram que outras soluções, com con- 
centrações acima e abaixo de 7,5\%, não apresentam vantagens $^{8}$, nem maior sobrevida ${ }^{9}$, mesmo quando se utilizam outros sais de sódio, outros cloretos e outras soluções sem eletrólitos ${ }^{10}$.

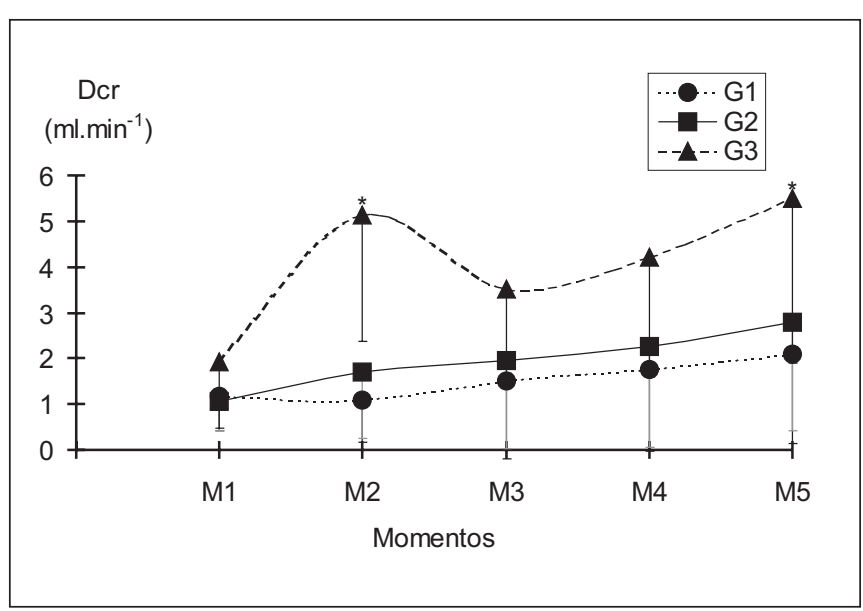

Figura 3 - Depuração de Creatinina $\left(\mathrm{ml}^{\mathrm{min}} \mathrm{mi}^{-1}\right)$. Média e Desvio Padrão em Cada Momento dos 3 Grupos

Comentário: Após a hemorragia (30\% da volemia) e posterior reposição (M1), a $\mathbf{D}_{\mathrm{cr}}$ apresentou valores superiores em relação aos dos demais grupos, e com relevância estatística ${ }^{*}(p<0,05)$ em G3*, no decorrer do experimento. Em cada momento, separadamente, G3* foi significativo somente em M2 e M5

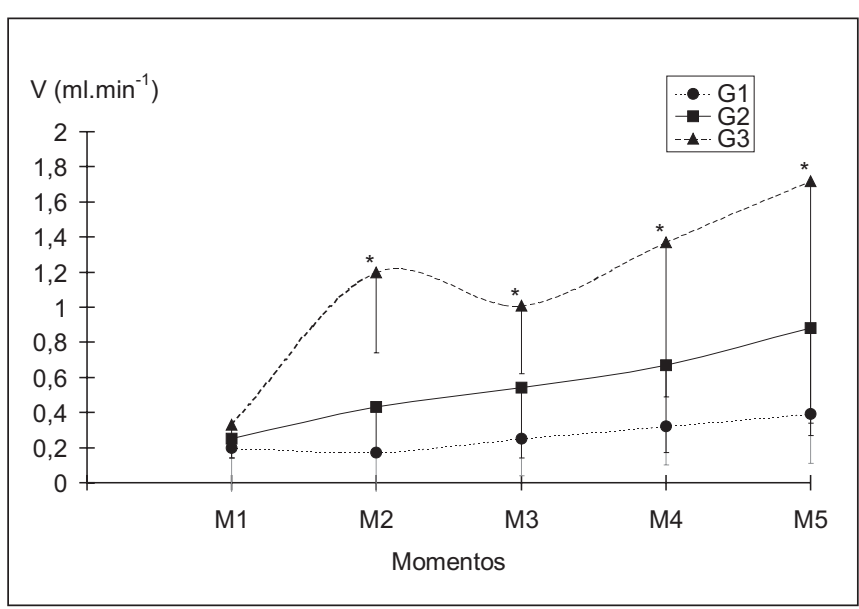

Figura 4 - Volume Urinário $\left(\mathrm{ml}_{\mathrm{m}} \mathrm{min}^{-1}\right)$. Média e Desvio Padrão em Cada Momento nos 3 Grupos

Comentário: Após a hemorragia ( $30 \%$ da volemia) e posterior reposição (M1), O volume urinário teve relevância estatística $(p<0,05)$ em G3*, com valores elevados, comparando-se aos dos demais grupos, no decorrer do experimento

A associação de dextran 70 a $6 \%$ permitiu prolongar os efeitos hemodinâmicos da SH por mais de 30 minutos $^{11}$. Na dose de $4 \mathrm{ml} . \mathrm{kg}^{-1}$ e utilizada uma única vez, a SHD é eficiente e virtualmente isenta de efeitos adversos ${ }^{12,13}$.
Em situações pós-hemorragia a injeção da SHD restaura o fluxo sangüíneo para vários órgãos, inclusive o rim ${ }^{14}$. Há meIhora do fluxo e reversão do estreitamento do leito capilar que se segue à hemorragia ${ }^{15,16}$. Essa propriedade da SHD, de restauração do fluxo capilar, tem implicações clínicas importantes no tratamento do choque hemorrágico ou de estados isquêmicos ${ }^{13}$.

A depuração de creatinina tem como tradução clínica o ritmo de filtração glomerular, que apresentou médias dos resultados, no grupo 3 , sempre bem superiores às dos demais grupos. Este momento, após hemorragia, é acompanhado de diminuição significativa no ritmo de filtração glomerular ${ }^{17}$ e a SHD mostrou-se capaz de não somente restaurar os valores iniciais, mas até ultrapassá-los. Em G2, a SH foi capaz do mesmo efeito, porém em menor escala, e os valores mostraram-se sempre muito próximos dos de G1, grupo que não recebeu nenhum tratamento.

Portanto, a SHD, principalmente devido à expansão do volume plasmático, aumenta a pressão arterial sistêmica, resultando na elevação da pressão hidrostática glomerular e do ritmo de filtração glomerular. Além disso, a SHD causa dilatação nas arteríolas glomerulares, por ação direta na musculatura lisa desses vasos, produzindo aumento da perfusão sangüínea e favorecendo ainda mais a filtração glomerular. Esse resultado representa um dos efeitos mais evidentes das soluções hipertônicas, que é o aumento do fluxo sangüíneo em vários órgãos ${ }^{18,19}$. O relaxamento na musculatura lisa dos vasos é efeito direto da hiperosmolaridade, sendo, nos vasos coronarianos, similar àquele produzido pela nitroglicerina ${ }^{20}$. Esta vasodilatação pré-capilar foi observada por vários autores ${ }^{6,21}$. A redução da viscosidade sangüínea associada à hemodiluição contribui ainda mais para a diminuição da resistência vascular periférica.

Osmolaridade é definida como uma expressão do número de partículas osmoticamente ativas que atraem a água através de membranas semipermeáveis até estabelecer-se o equilíbrio, por litro de solvente, enquanto que osmolalidade é a medida do número de partículas osmoticamente ativas por quilograma de solvente. Estima-se a osmolalidade pela seguinte fórmula: (sódio x 2) + (glicose/18) + (uréia/2,3) ${ }^{22}$.

Embora os osmóis por quilograma de água (osmolalidade) sejam os que determinam a pressão osmótica, para as soluções diluídas que formam os líquidos corporais, a diferença entre osmolalidade e osmolaridade é menor que 1\%. Este fato faz com que estes termos possam ser usados quase como sinônimos. Portanto, além de ser mais fácil expressar as concentrações dos líquidos corporais em relação a litros do que quilogramas de água, a prática em quase todos os estudos fisiológicos é utilizar osmolaridade em vez de osmolalidade.

Sabendo-se que a hiperosmolaridade ocorre quando a quantidade de partículas osmoticamente ativas eleva-se, tanto a uremia como a hipernatremia poderiam causar tal estado. Entretanto, como a uréia se distribui por toda a água corporal, somente um aumento do sódio plasmático é capaz de resultar em hipertonicidade, isto é, redistribuição, de ori- 
gem osmótica, de água do espaço intracelular para o extracelular $^{22}$.

A pressão osmótica de uma solução, em relação à do plasma, é comumente chamada de tonicidade. Portanto, um líquido com pressão osmótica similar à do plasma é chamado de isotônico. Soluções hipotônicas exercem baixa pressão osmótica e hipertônicas, como SH e SHD, alta pressão osmótica. A pressão osmótica é aproximadamente igual ao produto da osmolalidade ${ }^{22}$ por 19,3 , significando que cada mOsm gera pressão osmótica de $19,3 \mathrm{mmHg}$ a $37^{\circ} \mathrm{C}$ através de uma membrana semipermeável ideal ${ }^{3}$.

As soluções utilizadas neste experimento apresentam osmolaridade de aproximadamente $2400 \mathrm{mOsm} . \mathrm{I}^{-1}$, portanto sendo capazes de gerar pressão osmótica cerca de 8 vezes maior do que uma solução com osmolaridade próxima à do plasma. Geralmente, estas soluções induzem hiperosmolaridade moderada e temporária ${ }^{18}$, quando utilizadas nas doses recomendadas e em injeção única. Os limites superiores aceitáveis da osmolaridade e da concentração de sódio no plasma ainda não foram definidos ${ }^{23}$, mas raramente ocorrem problemas com osmolaridades menores que 350 mOsm. I $^{-1}$, número este não alcançado na maioria dos experimentos ${ }^{6,24}$. Relatos sobre altos valores de osmolaridade geralmente estão ligados a doses elevadas ou injeções repetidas $^{25}$.

Por isso, nos grupos 2 e 3 observou-se elevação das depurações osmolar e de sódio, que indica maior perda de substâncias osmoticamente ativas pela urina e natriurese, provavelmente provocada pela inibição da secreção de aldosterona. Esta inibição, induzida pela administração de SH e SHD, deve ocorrer devido à rápida correção da hipovolemia e da instalação de moderada hiperosmolaridade plasmática.

Richards e col. ${ }^{26}$ sugeriram que a liberação do hormônio natriurético atrial seria responsável por esta magnitude de aumento na diurese. No entanto, não foi encontrada elevação dos níveis séricos do peptídeo natriurético atrial em reposta à injeção de SHD ${ }^{27,28}$.

Essa diurese desproporcionalmente maior que o volume administrado, após a injeção de SH e SHD, ocorre mesmo em presença de hipovolemia e concentrações circulantes elevadas de hormônio antidiurético. A produção elevada de urina pode até causar prejuízos pela perda líquida que ocasiona e pode ser o fator responsável pelos efeitos hemodinâmicos transitórios das soluções hipertônicas ${ }^{29}$.

Para Rocha e Silva ${ }^{18}$, as soluções hipertônicas interferem com as secreções endócrinas, em animais com hipotensão pós-hemorrágica, porque produzem correção rápida da hipovolemia, o que pode determinar diminuição das concentrações circulantes de vasopressina, renina e angiotensina

30. A redução da vasopressina circulante aparece, segundo os autores, apesar do aumento da osmolaridade que, normalmente, é responsável pela sua secreção. Nesta situação, a resposta à osmolaridade é sobreposta pela correção da hipovolemia, que é fator secretório de vasopressina ${ }^{18}$. Portanto, soluções hipertônicas determinam incremento significativo na diurese, que se mantém por horas após a injeção inicial ${ }^{15}$.
Observa-se semelhança entre os três grupos, no que se refere aos valores de depuração de água livre e osmolaridade urinária, e esse fato demonstra que os grupos que receberam SH e SHD não apresentaram comportamento diferente do controle, apesar de apresentarem diurese mais elevada. Portanto, a osmolaridade urinária mostrou redução significativa com o tempo, mas isso não foi determinado pela SH ou SHD, já que ocorreu o mesmo em todos os grupos. Com a depuração de água livre, que representa a excreção de água livre de solutos, não houve diferença significativa em nenhum momento ou grupo.

Adepuração osmolar corresponde ao volume de plasma que é liberado de todas as substâncias osmoticamente ativas na unidade de tempo, ou seja, eqüivale à depuração de íons. Logo, houve incremento significativo na depuração de íons em G3, devido à administração de SHD. Esse fato foi reconhecido pelo aumento na curva de depuração osmolar que ocorreu inicialmente em M2, momento imediatamente após a injeção de SHD. Em seguida, houve pequena queda, mas, ainda assim, manteve-se em patamar significativamente elevado em relação aos demais grupos. Por outro lado, em $\mathrm{G} 2$, a injeção de SH determinou aumento da depuração de íons, não significativo, mas de importância biológica.

O aumento observado nos grupos 2 e 3 , que receberam soluções hipertônicas de sódio, ocorreu, possivelmente, devido à interferência dessas soluções na secreção de vasopressina e aldosterona. A redução dos níveis sangüíneos de aldosterona ocasionou maior depuração de substâncias osmoticamente ativas e de sódio, assim como a redução de vasopressina determinou maior perda de água livre de solutos pela urina, nesses grupos.

A administração de SH e SHD, que contêm carga elevada de sódio, foi responsável pelo aumento da concentração plasmática deste íon. Esta concentração não se altera quando a expansão é realizada com soluções isotônicas, devido aos mecanismos renais que levariam à maior excreção e menor reabsorção do sódio em todos os segmentos do néfron, contanto que estejam íntegros. No entanto, foram administradas duas soluções hipertônicas, que exercem maior pressão osmótica que o plasma, e cuja concentração elevada de sódio ultrapassa essa capacidade de excreção rápida, determinando aumento da concentração desse íon no plasma. Tal fato foi observado neste experimento, em M2, que é o momento iniciado logo após a reanimação hipertônica, isto é, com a infusão de SH e SHD, e, portanto, o momento mais susceptível a aumento significativo das concentrações plasmáticas do sódio. Na dosagem de $4 \mathrm{ml} . \mathrm{kg}^{-1}$ e administração única, a hipernatremia observada é moderada e transitória 31.

Assim como na depuração de sódio, G3 teve destacadamente valores médios mais altos na curva de excreção urinária, demonstrando maior capacidade da SHD em elevar os valores séricos de sódio, assim como sua depuração.

Segundo Vincent ${ }^{32}$, o desenvolvimento de hipocalemia é comumente observado e provavelmente determinado pelo aumento da perda de potássio na urina, podendo, inclusive, causar disritmias, particularmente em pacientes que fazem 
uso de digital. Níveis séricos diminuídos de potássio também foram observados por outros autores ${ }^{2,16,33,34}$. As causas dessa redução são a ausência de potássio nessas soluções e a rápida expansão do fluido extracelular, que produzem diluição do potássio extracelular.

Neste estudo, observou-se claramente aumento da excreção de potássio com o decorrer do experimento.

Mesmo seguindo-se todos os passos adequados para identificar qualquer alteração renal visível à microscopia óptica, não foi evidenciada lesão compatível com necrose tubular aguda após isquemia, a qual, entretanto, foi evidenciada em relatos anteriores por vários autores ${ }^{35,36}$. A presença de necrose tubular aguda pós-isquêmica poderia ser visível ao microscópio óptico, ao menos em G1, se o período entre o fim do experimento e a retirada do órgão para estudo histopatológico tivesse sido maior, porque, então, poderia haver tempo suficiente para a delimitação da necrose.

Após a análise estatística dos resultados obtidos nos três grupos experimentais, podemos concluir que a solução hipertônica de cloreto de sódio a 7,5\% em dextran 70 a $6 \%$, administrada logo após episódio de hemorragia moderada e 15 minutos antes de insulto isquêmico de 30 minutos, foi mais eficiente que a solução hipertônica de cloreto de sódio a $7,5 \%$ em proteger o rim das repercussões da isquemia-reperfusão em cães.

\section{Efficacy of $7.5 \%$ Hypertonic Sodium Chloride, with and without 6\% Dextran 70, in Renal Function Preservation of Hypovolemic Dogs Submitted to Ischemia-Reperfusion}

Geraldo Rolim Rodrigues Júnior, TSA, M.D., José Luiz Gomes do Amaral, TSA, M.D., Yara Marcondes Machado Castiglia, TSA, M.D., Mariangela Esther Alencar Marques, M.D.

\section{INTRODUCTION}

Low doses of hypertonic sodium chloride promptly restore intravascular volume due to water redistribution as from the intracellular compartment. This blood volume increase is transient, but it is earlier observed as compared to isosmotic fluids infusion in the same time period. In addition, such solutions may improve performance of several organs, including the heart, be it by contractility effects, afterload decrease or even for removing myocardial oxygen free radicals. The latter effect is produced by dextran 70 when associated to $7.5 \%$ sodium chloride ${ }^{1}$. Although improving perfusion of several organs, including the kidney, such solutions was not widely used due to the short duration of their beneficial effects. Currently, low volumes of $7.5 \%$ hypertonic sodium chloride for treating hypovolemia, hypotension and shock became very popular with the association of $6 \%$ dextran 70 . This for- mulation has prolonged solutions effects and maximized beneficial physiological effects of both components ${ }^{1}$. Several animal studies have shown the efficacy of such solutions in attenuating or even reverting many abnormalities caused by hemorrhagic shock and hypovolemia. When facing such situations a major concern is renal preservation because its function, which is fundamental for homeostasis, may be severely affected reaching different failure degrees and impairing patient's recovery.

Transient renal blood flow interruption causes anatomic and physiological changes which are more severe as a function of ischemia duration. A30-minute ischemia associated to previous hypovolemia will produce severe renal injury in the absence of treatment or prevention.

Hypertonic sodium chloride, associated or not to hyperoncotic colloids, may be effective in protecting kidneys from such foreseeable situations. Several authors have suggested that the so-called hypertonic resuscitation from hemorrhagic shock certainly reverts renal dysfunction ${ }^{2}$. Other investigators have stated that $7.5 \%$ sodium chloride associated to dextran 70 may revert some noxious ischemia or ische$\mathrm{mia} /$ reperfusion-induced effects, especially those caused by capillary perfusion increase ${ }^{3}$.

Our study aimed at analyzing the real benefits of $7.5 \%$ hypertonic sodium chloride (HS), associated or not to $6 \%$ dextran 70 (HSD) on the simulation of abnormal renal function in dogs, caused by hypovolemia associated to 30 -minute ischemia.

\section{METHODS}

After the Experimental Research Ethics Committee approval, 24 adult mixed-breed dogs with undefined ages, from both sexes and weighing 7 to $33 \mathrm{~kg}$ were studied. Animals were anesthetized with $30 \mathrm{mg} \cdot \mathrm{kg}^{-1}$ intravenous sodium pentobarbital (SP) for induction and $5 \mathrm{mg} \cdot \mathrm{kg}^{-1}$ for maintenance (105 minutes after priming dose).

Animals were randomly distributed in 3 experimental groups of 8 dogs.

Group 1 (G1) - Animals were submitted to right lombotomy for right kidney removal and left lombotomy for ureter catheterization and left renal artery clamping for 30 minutes. All animals were submitted to approximately $29 \%$ to $30 \%$ hemorrhage, enough to decrease circulating volume and cause hypovolemia, but not shock.

Group 2 (G2) - The same as G1, followed by intravenous administration of $4 \mathrm{ml}^{\mathrm{kg}} \mathrm{kg}^{-1}$ of HS in 3 minutes, 15 minutes after scheduled hemorrhage.

Group 3 (G3) - The same as G1, followed by intravenous administration of HSD in the same dose and speed used for $\mathrm{G} 2$.

After a 14-hour fast, animals were anesthetized with sodium pentobarbital and placed in the supine position in a Claude Bernard trough. The following procedures were performed:

1. Tracheal intubation and air controlled ventilation using a K. Takaoka, Mod 850-10 anesthesia machine. Tidal 
volume was $15 \mathrm{ml}_{\mathrm{kg}} \mathrm{kg}^{-1}$ and respiratory rate was $15 \mathrm{mo}-$ vements per minute;

2. Left femoral vein dissection and catheterization for inferior vena cava pressure monitoring with a water pressure gage, initial $0.2 \mathrm{mg} \cdot \mathrm{kg}^{-1}$ sodium pentobarbital and alcuronium administration and then $0.006 \mathrm{mg}^{\mathrm{kg}}{ }^{-1}$ for additional doses, venous blood collection, biochemical dosing and lactated Ringer's continuous infusion (1 $\left.\mathrm{ml} . \mathrm{kg}^{-1} \cdot \mathrm{min}^{-1}\right)$; right femoral artery dissection and catheterization for mean blood pressure monitoring with a mercury pressure gage and left femoral artery dissection and catheterization for $20 \mathrm{ml} . \mathrm{kg}^{-1}$ blood volume removal;

3. Left femoral vein dissection and catheterization for $\mathrm{PAH}$ priming dose $\left(4 \mathrm{mg} \cdot \mathrm{kg}^{-1}\right)$ and creatinine $\left(30 \mathrm{mg} \cdot \mathrm{kg}^{-1}\right)$ in lactated Ringer's, 30 minutes after Ringer's solution continuous infusion. Next, continuous PAH (0.06 g\%) and creatinine $(0.15 \mathrm{~g} \%)$ in Ringer's solution infusion was installed and maintained until the end of the experiment in the dose of $0.6 \mathrm{mg}$ creatinine and $0.24 \mathrm{mg} \mathrm{PAH}$ per minute, per animal's kilo $\left(0.4 \mathrm{ml} . \mathrm{kg}^{-1}\right)$;

4. Right lombotomy for right kidney removal and left lombotomy for ureter catheterization and left renal peduncle dissection; left renal artery exposure, non-traumatic clamp insertion which would remain open until clamping time;

5. Ureter catheterization with 6 or 8 polivynil probe, depending on the animal. Urine, whenever produced, would continuously flow through the probe during the experiment;

6.Placement of rectal alcohol thermometer for temperature readings;

7. Removal of left femoral artery blood 30 minutes after priming injection;

8. Injection of $0.9 \%$ sodium chloride in $\mathrm{G} 1,7.5 \%$ in $\mathrm{G} 2$ and $7.5 \%$ in $6 \%$ dextran 70 in G3, 15 minutes after hemorrhage and 45 minutes after priming injection;

9. Left renal artery clamping in all groups and unclamping 30 minutes later;

10. Animals' euthanasia with $20 \%$ intravenous formalin and left kidney removal for histopathologic study.

Attributes were divided in two groups.

a) to control homogeneity: demographics - animal weight $(\mathrm{kg})$, sex and length $\left(\mathrm{m}^{-1}\right)$; respiratory rate $\left(\mathrm{mov} \cdot \mathrm{min}^{-1}\right)$, tidal volume $\left(\mathrm{ml}^{\mathrm{kgg}}{ }^{-1}\right)$, hematocrit $(\mathrm{Ht})$ $(\%)$ and rectal temperature $\left({ }^{\circ} \mathrm{C}\right)$;

b) to meet research goals: hemodynamic: mean blood pressure (MBP), inferior vena cava pressure (ICP); heart rate $(\mathrm{HR})$; renal plasma flow (measured by PAH clearance $-\mathrm{C}_{\mathrm{PAH}}=\mathrm{U}_{\mathrm{PAH}} \times \mathrm{V} / \mathrm{P}_{\mathrm{PAH}}$, where $\mathrm{V}=$ urine output, $\mathrm{U}$ and $\mathrm{P}=$ urine and plasma $\mathrm{PAH}$ concentration, respectively; renal blood flow $\left(\mathrm{RBF}=\mathrm{C}_{\mathrm{PAH}} / 1-\mathrm{Ht}\right)$; renal vascular resistance (RVR $=M B P / R B F)$. Renal function: glomerular filtration rate (GFR) (measured by creatinine clearance $-\mathrm{C}_{\mathrm{cr}}=\mathrm{U}_{\mathrm{cr}} \times \mathrm{V} / \mathrm{P}_{\mathrm{cr}}$ ); urine out- put $(\mathrm{V})$; glomerular filtration $\left(\mathrm{FF}=\mathrm{C}_{\mathrm{Cr}} / \mathrm{C}_{\mathrm{PAH}}\right)$; plasma osmolarity $\left(\mathrm{P}_{\mathrm{osm}}\right)$; urine osmolarity $\left(\mathrm{U}_{\mathrm{osm}}\right)$; osmolar clearance $\left(\mathrm{C}_{\mathrm{osm}}=\mathrm{U}_{\mathrm{osm}} \times \mathrm{V} / \mathrm{P}_{\mathrm{osm}}\right)$; sodium and potassium clearance $\left(\mathrm{C}_{\mathrm{Na} \text { or } \mathrm{K}}=\mathrm{U}_{\mathrm{Na} \text { or K }} \times \mathrm{V} / \mathrm{P}_{\mathrm{Na} \text { or } \mathrm{K}}\right)$; free water clearance $\left(\mathrm{C}_{\mathrm{H}_{2} \mathrm{O}}=\mathrm{V}-\mathrm{C}_{\mathrm{osm}}\right)$; sodium urinary excretion $\left(\mathrm{U}^{+}{ }_{\text {nu }} \times \mathrm{V}=\mathrm{U}_{\mathrm{Na}} \times \mathrm{V}\right)$; sodium fractionated excretion ( $\mathrm{EF}_{\mathrm{Na}}=\mathrm{C}_{\mathrm{Na}} / \mathrm{C}_{\mathrm{cr}} \times 100$ ); potassium urinary excretion $\left(\mathrm{U}_{\mathrm{K}} \mathrm{V}=\mathrm{K}_{\mathrm{u}}^{+} \times \mathrm{V}\right)$; potassium fractionated excretion (EF $\left.=D_{K} / C_{c r} \times 100\right)$. Left kidney histopathologic study.

These parameters were obtained in the following moments and results were compared among groups. This study has not aimed at measuring hemodynamic variables soon after hemorrhage; rather the aim was evaluating the efficacy of some solutions. To determine the impact, G1 was studied as the control group.

M1 and M2 - obtained 15 and 60 minutes after PAH and creatinine priming injection;

M3 and M4 - obtained 105 and 120 minutes after PAH and creatinine priming injection (immediately and 15 minutes after unclamping, respectively); and M5 - 135 minutes after PAH and creatinine priming injection and 30 minutes after unclamping.

Groups $\mathrm{G} 2$ and $\mathrm{G} 3$ received $4 \mathrm{ml} . \mathrm{kg}^{-1}$ of animal's weight of HS and HSD, respectively, for hemorrhage replacement $15 \mathrm{mi}-$ nutes after blood removal. G1 received the same dose of $0.9 \%$ sodium chloride

Chart I - Drugs and Solutions Administered During the Experiment

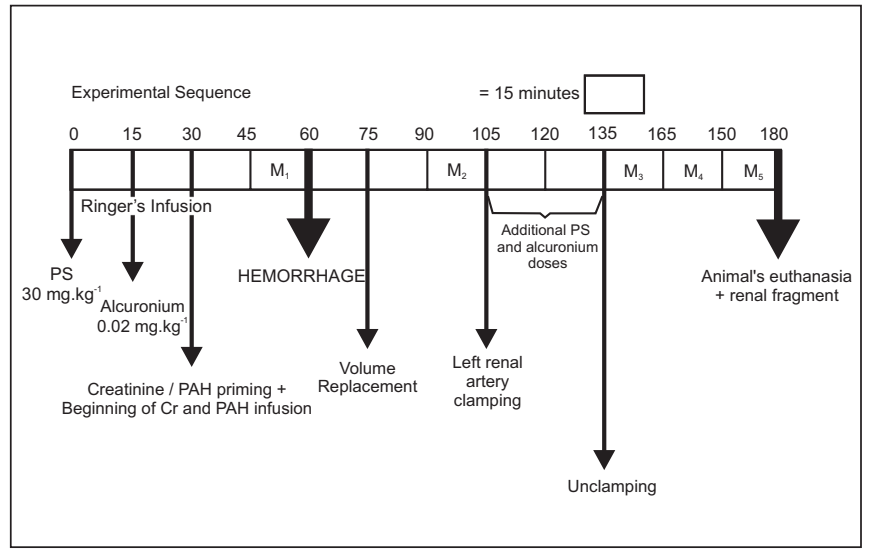

Histopathological samples were placed in $5 \%$ formalin. After being fixed for more than 48 hours, they were cleaned and placed in wax to be further cut and dyed with hematoxylin-eosin and Schiff's periodic acid (SPA). Samples were labeled and analyzed by an investigator blind to the experimental group they belonged to.

Morrison's profile analysis was used for all variables and $p$ was determined as 0.05 or $5 \%(\alpha<$ or $=0.05)$; a star $\left({ }^{*}\right)$ was used to highlight significant values. Significance trend was referred when $0.05<p<0.10$. Tukey's method was used to compare groups means, by calculating the minimum signifi- 
cant difference for $\alpha=0.05$. Demographics were compared by analysis of variance (ANOVA).

\section{RESULTS}

Our most significant results are shown in tables I, II and III and figures 1, 2, 3 and 4 .

There has been homogeneity (ANOVA) in weight, length and sex among dogs of the three experimental groups.

$\mathrm{U}_{\text {osm }}, \mathrm{C}_{\mathrm{H}_{2} \mathrm{O}}, \mathrm{EF}_{\mathrm{na}}, \mathrm{EF}_{\mathrm{K}}, \mathrm{PaO}_{2}, \mathrm{PaCO}_{2}, \mathrm{pH}, \mathrm{PCl}, \mathrm{P}_{\text {osm }}, \mathrm{FF}, \mathrm{V}$, $\mathrm{C}_{\text {osm }}, \mathrm{C}_{\mathrm{NA}}, \mathrm{U}_{\mathrm{NA}} \mathrm{V}, \mathrm{C}_{\mathrm{K}}$ and $\mathrm{U}_{\mathrm{K}} \mathrm{V}$ were not significantly different within groups and moments studied.
Rectal temperature showed uniform and continuous decrease along the trial in all groups.

Even following every adequate step to identify any lesion visible at optical microscopy, no lesion compatible with acute tubular necrosis was observed.

\section{DISCUSSION}

The anesthetic technique used is standard for dogs due to its proven efficacy in reaching an adequate anesthetic depth in a few minutes and for not affecting renal function.

Table I - Renal Blood Flowl (RBF) and Renal Vascular Resistance (RVR). Mean and Standard Deviation in each Moment for the 3 Groups

\begin{tabular}{|c|c|c|c|c|c|c|c|}
\hline \multirow[t]{2}{*}{ Parameter } & \multirow[t]{2}{*}{ Groups } & & \multicolumn{3}{|c|}{ Moments } & \multicolumn{2}{|r|}{ Analysis } \\
\hline & & M1 & M2 & M3 & M4 & M5 & \\
\hline \multirow{3}{*}{$\begin{array}{l}\text { RBF } \\
\left(\mathrm{ml}^{\left.\mathrm{min}-{ }^{1}\right)}\right)\end{array}$} & G1 & $5.73 \pm 3.39$ & $2.71 \pm 1.68$ & $3.08 \pm 3.49$ & $3.85 \pm 3.42$ & $4.93 \pm 4.39$ & \multirow{3}{*}{$\begin{array}{l}\text { Non-similar profiles } \\
(\mathrm{G} 1=\mathrm{G} 2)<\mathrm{G} 3-\mathrm{M} 2 \\
(\mathrm{G} 1=\mathrm{G} 2)<\mathrm{G} 3-\mathrm{M} 3 \\
(\mathrm{G} 1=\mathrm{G} 2)<\mathrm{G} 3-\mathrm{M} 4 \text { and } \\
(\mathrm{G} 1=\mathrm{G} 2)<\mathrm{G} 3(\mathrm{M} 5) \mathrm{p}<0.05\end{array}$} \\
\hline & G2 & $5.61 \pm 2.55$ & $5.62 \pm 3.78$ & $5.82 \pm 3.21$ & $7.10 \pm 5.09$ & $8.80 \pm 5.76$ & \\
\hline & $\mathrm{G}^{*}$ & $8.85 \pm 8.34$ & $20.23 \pm 12.10$ & $12.25 \pm 6.78$ & $17.03 \pm 14.63$ & $18.66 \pm 11.38$ & \\
\hline \multirow{3}{*}{$\begin{array}{l}\text { RVR; }\left(\mathrm{mmH}^{-1}\right) \\
\left(\mathrm{ml}^{-1} \cdot \mathrm{min}^{-1}\right)\end{array}$} & G1 & $36.12 \pm 36.23$ & $50.19 \pm 31.51$ & $80.20 \pm 63.88$ & $51.65 \pm 45.18$ & $51.42 \pm 71.84$ & \multirow{3}{*}{$\begin{array}{l}\text { Similar profiles } \\
\text { (trend to interaction) } \\
\text { p<0.05 } \\
\text { Profiles in different levels } \\
\mathrm{G} 1>\mathrm{G} 2>\mathrm{G} 3 \\
0.05<\mathrm{p}<0.1\end{array}$} \\
\hline & G2 & $28.30 \pm 12.62$ & $41.91 \pm 31.75$ & $32.89 \pm 23.83$ & $43.75 \pm 57.22$ & $25.13 \pm 20.00$ & \\
\hline & $\mathrm{G}^{*}$ & $23.77 \pm 15.60$ & $9.28 \pm 6.17$ & $13.58 \pm 7.51$ & $11.86 \pm 7.97$ & $10.12 \pm 6.51$ & \\
\hline
\end{tabular}

Comment: As to RBF. only G3* showed statistical significance and higher volumes than other groups during the experiment. There was a decreased RVR being higher in the control group (G1) and lower in $\mathrm{G}^{*}$ (trend to interaction), which had the lowest resistance among groups

Table II - Sodium Attributes ( $\left.\mathrm{Na}^{+}\right)$- Plasma, Urine Clearance and Excretion - Osmolar Clearance. Mean and Standard Deviation of Values in each Moment in All Groups

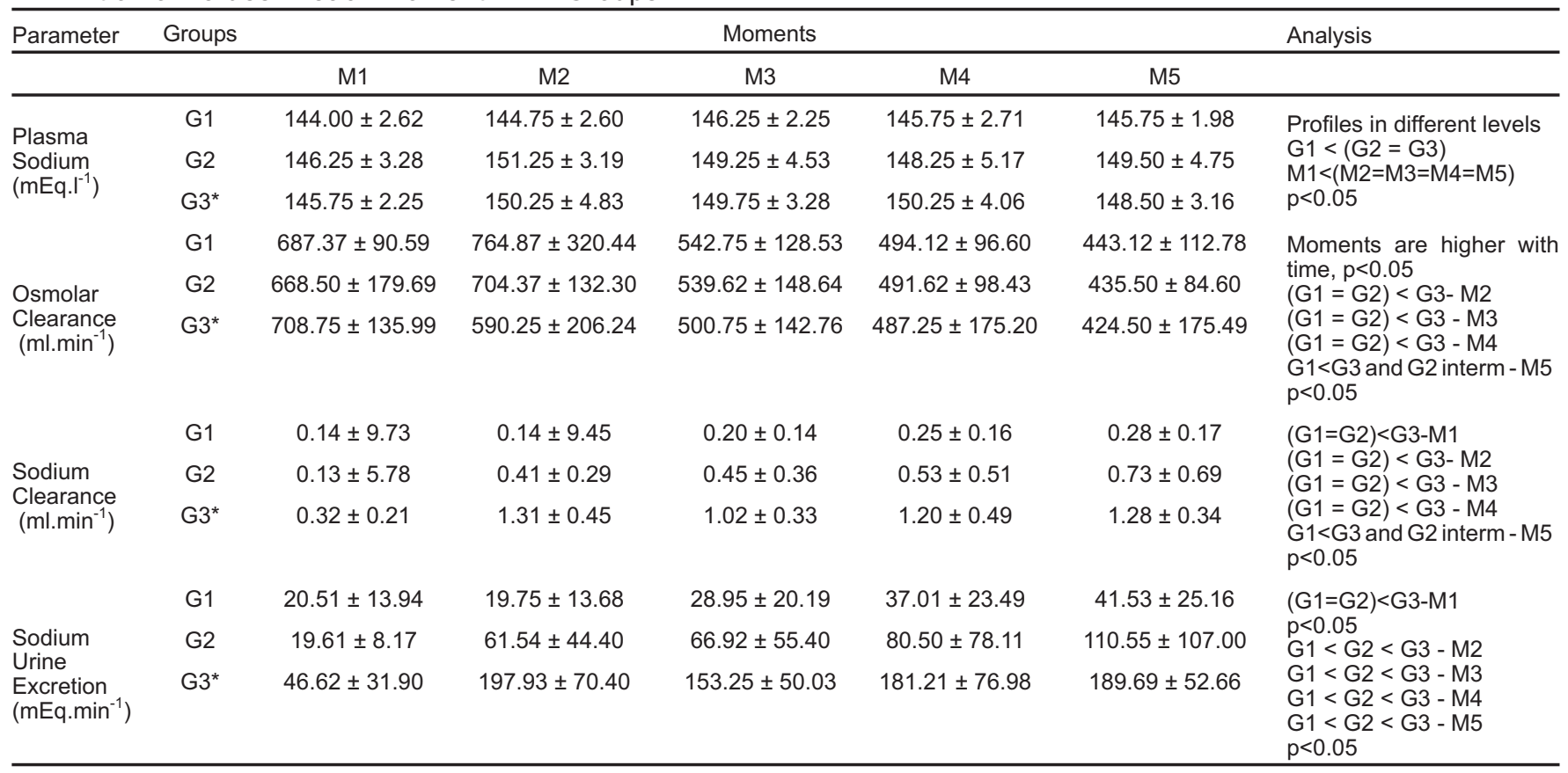

Comment: $\mathrm{Na}^{+}$was present in all variables since this ion is directly responsible for osmotic pressure. Solutions administered in $\mathrm{G} 3$ and $\mathrm{G} 2$ determined increased plasma $\mathrm{Na}^{+}$in those groups as compared to control. Both $\mathrm{Na}^{+}$urine clearance and excretion were more markedly increased in $\mathrm{G}^{*}$ throughout the experiment 
EFFICACY OF 7.5\% HYPERTONIC SODIUM CHLORIDE, WITH AND WITHOUT 6\% DEXTRAN 70, IN RENAL FUNCTION PRESERVATION OF HYPOVOLEMIC DOGS SUBMITTED TO ISCHEMIA-REPERFUSION

Table III - Potassium Attributes $\left(\mathrm{K}^{+}\right)$, Urine Clearance and Excretion. Mean and Standard Deviation in each Moment for All Groups

\begin{tabular}{|c|c|c|c|c|c|c|c|}
\hline \multirow[t]{2}{*}{ Parameter } & \multirow[t]{2}{*}{ Groups } & & \multicolumn{3}{|c|}{ Moments } & \multicolumn{2}{|r|}{ Analysis } \\
\hline & & M1 & M2 & M3 & M4 & M5 & \\
\hline \multirow{3}{*}{$\begin{array}{l}\text { Potassium } \\
\text { clearance } \\
\left(\mathrm{ml}_{\mathrm{min}}^{-1}\right)\end{array}$} & G1 & $2.87 \pm 1.57$ & $3.17 \pm 2.08$ & $2.95 \pm 2.40$ & $3.87 \pm 2.96$ & $3.40 \pm 2.51$ & \multirow{3}{*}{$\begin{array}{l}(\mathrm{G} 1=\mathrm{G} 2)<\mathrm{G} 3-\mathrm{M} 2 \\
\mathrm{G} 1<\mathrm{G} 2<\mathrm{G} 3-\mathrm{M} 3 \\
\mathrm{G} 1<\mathrm{G} 2<\mathrm{G} 3-\mathrm{M} 4 \\
\mathrm{G} 1<\mathrm{G} 3<\mathrm{G} 2-\mathrm{M} 5 \\
\text { Moments increased with } \\
\text { time = G2-G3 } \\
\mathrm{p}<0.05\end{array}$} \\
\hline & G2 & $4.47 \pm 2.76$ & $5.87 \pm 3.58$ & $6.19 \pm 2.75$ & $8.44 \pm 4.74$ & $10.04 \pm 4.38$ & \\
\hline & G3* & $5.35 \pm 1.87$ & $9.30 \pm 2.06$ & $10.30 \pm 1.46$ & $13.89 \pm 4.28$ & $13.41 \pm 2.92$ & \\
\hline \multirow{3}{*}{$\begin{array}{l}\text { Potassium } \\
\text { urine excretion } \\
\left(\mathrm{mEq} \cdot \mathrm{min}^{-1}\right)\end{array}$} & G1 & $11.11 \pm 6.15$ & $11.77 \pm 8.24$ & $10.81 \pm 9.44$ & $14.06 \pm 11.73$ & $14.09 \pm 9.75$ & \multirow{3}{*}{$\begin{array}{l}\mathrm{G} 1<\mathrm{G} 3 \text { and } \mathrm{G} 2 \text { interm- M2 } \\
\mathrm{G} 1<\mathrm{G} 2<\mathrm{G} 3-\mathrm{M} 3 \\
\mathrm{G} 1<\mathrm{G} 2<\mathrm{G} 3-\mathrm{M} 4 \\
\mathrm{G} 1<(\mathrm{G} 2=\mathrm{G} 3)-\mathrm{M} 5 \\
\mathrm{p}<0.05\end{array}$} \\
\hline & G2 & $16.91 \pm 10.81$ & $21.71 \pm 16.68$ & $23.68 \pm 11.00$ & $32.49 \pm 19.09$ & $38.74 \pm 17.14$ & \\
\hline & G3* & $19.96 \pm 6.51$ & $32.48 \pm 6.41$ & $39.05 \pm 5.92$ & $53.75 \pm 18.50$ & $49.16 \pm 9.92$ & \\
\hline
\end{tabular}

Comment: Groups receiving hypertonic solutions (more marked in $\mathrm{G}^{*}$ ) showed higher $\mathrm{K}^{+}$urine clearance and excretion (markedly in $\mathrm{G} 3^{*}$ ) as compared to control (G1) throughout the experiment. However, plasma $\mathrm{K}^{+}$did not show differences

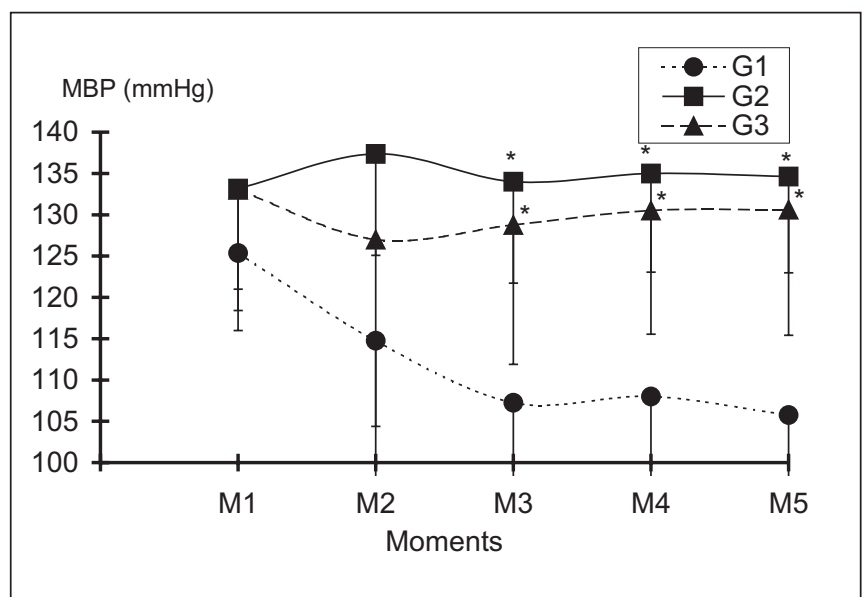

Figure 1 - Mean Blood Pressure ( $\mathrm{mmHg}$ ). Mean and Standard Deviation in each Moment in All Groups

Comment: After hemorrhage (30\% of volume replacement) and reperfusion (M1), MBP significantly and progressively decreased in $\mathrm{G} 1 *(\mathrm{p}<0.05)$ (control) throughout the experiment as compared to other groups ${ }^{*}(p<0.05)$, showed the highest $\mathrm{MBP}$ in M3, M4 e M5

The acute renal ischemia induced in dogs followed a model already developed in a previous study, but this time it was associated to hypovolemia caused by the removal of approximately $29 \%$ blood volume, which is a method proved to cause hypovolemia and widely used by several authors ${ }^{5}$.

Renal function impact determined by the association of hypovolemia and acute ischemia was observed in group 1 . In groups 2 and 3 an effective renal function protection against the noxious effects of this association was tested with HS and HSD.

The effects of HS were determined in 1980 by Velasco et al. ${ }^{6}$, who observed that $7.5 \%$ hypertonic sodium chloride, with an osmolarity of approximately $2,400 \mathrm{mOsm}^{-1}$ and in the dose of $4 \mathrm{ml}^{\mathrm{kg}}{ }^{-1}$ would induce maximum plasma expansion at moment zero, similarly to $883 \mathrm{mOsm} . \mathrm{I}^{-1}$ in a dose of $12 \mathrm{ml} . \mathrm{kg}^{-1}$. Several authors have shown that other solutions, in concen-

Revista Brasileira de Anestesiologia

Vol. 52, № 1, Janeiro - Fevereiro, 2002 trations above and below $75 \%$, had neither advantages ${ }^{8}$ nor increased survival ${ }^{9}$ even when different sodium salts, chlorides and other electrolyte-free solutions were used ${ }^{10}$.

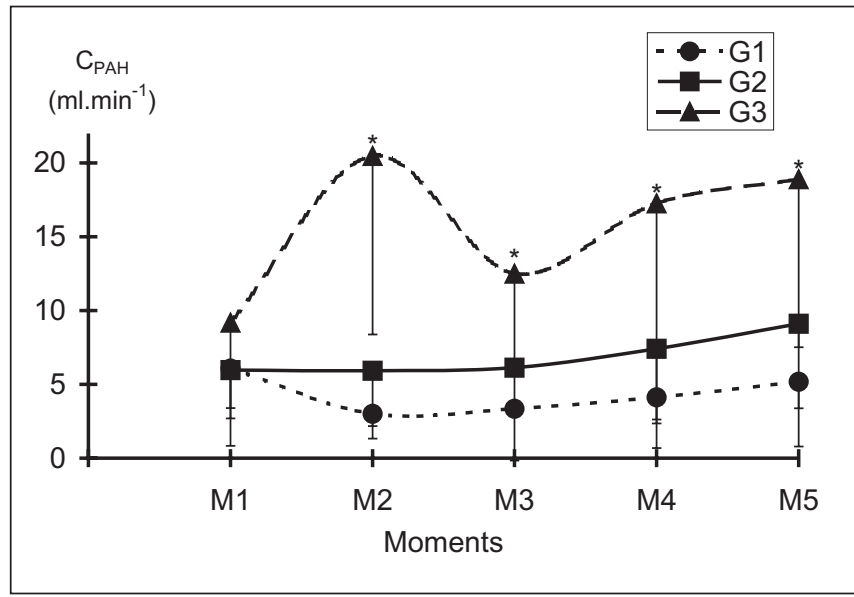

Figure 2 - PAH Clearance (Effective Plasma Renal Flow) (ml. $\mathrm{min}^{-1}$ ) Mean and Standard Deviation in each Moment in All Groups Comment: After hemorrhage (30\% of volume replacement) and reperfusion (M1), $C_{\text {PAH }}$ was higher only in $G 3^{*}(p<0.05)$, throughout the experiment

The association of $6 \%$ dextran 70 has prolonged HS hemodynamic effects for more than 30 minutes ${ }^{11}$. In a single dose of 4 $\mathrm{ml} . \mathrm{kg}^{-1}, \mathrm{HSD}$ is effective and virtually free of adverse effects 12,13

In post-hemorrhagic situations, HSD restores blood flow to several organs, including the kidney ${ }^{14}$. There is flow improvement and recovery of post-hemorrhage capillary bed narrowing ${ }^{15,16}$. This capillary flow recovery ability of HSD has important clinical implications in treating hemorrhagic shock or ischemia $^{13}$.

The clinical translation of creatinine clearance is glomerular filtration rate which, in group 3 showed mean results always well above the other groups. This post-hemorrhage moment 
is followed by a significant glomerular filtration rate decrease ${ }^{17}$ and HSD was able not only to restore initial values but also to go beyond them. In G2, HS produced the same effect, but in a lesser degree and values were always very close to $\mathrm{G} 1$, which was the group receiving no treatment.

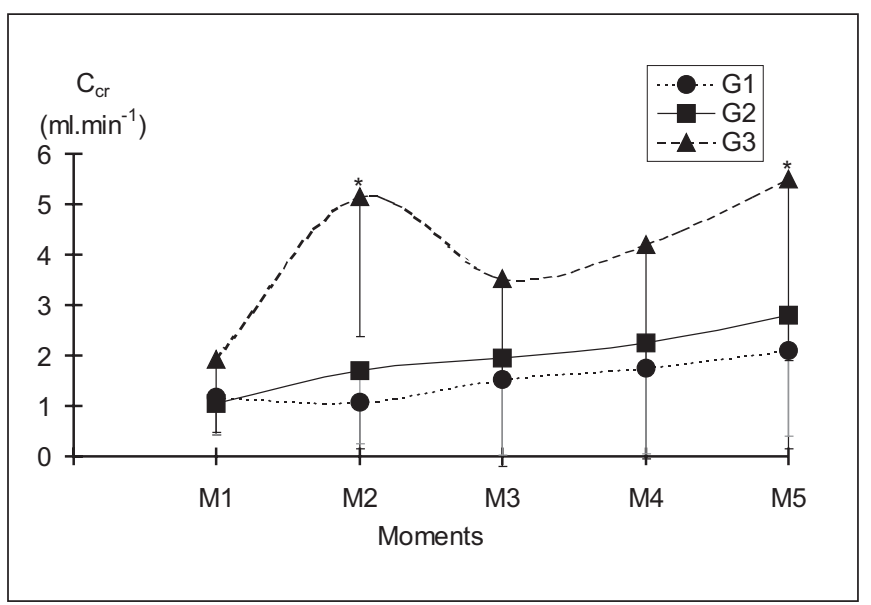

Figure 3 - Creatinine Clearance $\left(\mathrm{ml} \cdot \mathrm{min}^{-1}\right)$. Mean and Standard Deviation in each Moment for All Groups

Comment: After hemorrhage (30\% of volume replacement) and reperfusion (M1), $\mathbf{C}_{\mathrm{cr}}$ was higher as compared to other groups, and with statistical relevance * $(p<0.05)$ in $\mathrm{G}^{*}$, throughout the experiment. Separately, in each moment, G3* was significant only in M2 and M5

So, HSD, especially due to plasma volume expansion, increases systemic blood pressure resulting in an increase in glomerular hydrostatic pressure and glomerular filtration rate. In addition, HSD dilates glomerular arterioles by directly acting on their smooth muscles causing an increase in blood perfusion and further favoring glomerular filtration.

This is one of the most apparent results of hypertonic solutions, that is, blood flow increase in several organs ${ }^{18,19}$. Vessels smooth muscle relaxation is a direct hyperosmolarity effect, which is similar to the effect of nitroglycerin on coronary vessels ${ }^{20}$. This pre-capillary vasodilation has been observed by several authors ${ }^{6,21}$. Hemodilution-related blood viscosity decrease contributes even further for peripheral vascular resistance decrease.

Osmolarity is defined as an expression of the number of osmotically active particles which attract water through semi-patent membranes until a balance is attained per liter of solvent, while osmolality is a measurement of the number of osmotically active particles per kilogram of solvent. Osmolality is estimated by the following formula (sodium $\times 2)+($ glucose/18) + (urea/2.3) ${ }^{22}$

Although osmols per kilo of water (osmolality) are those determining osmotic pressure, the difference between osmolality and osmolarity for diluted solutions forming body fluids is less than $1 \%$. This allows both terms to be used almost as synonyms. So, in addition to be easier to express body fluids concentration as a function of liters and not of kilograms of water, the practice in almost all physiologic studies is to use osmolarity and not osmolality.

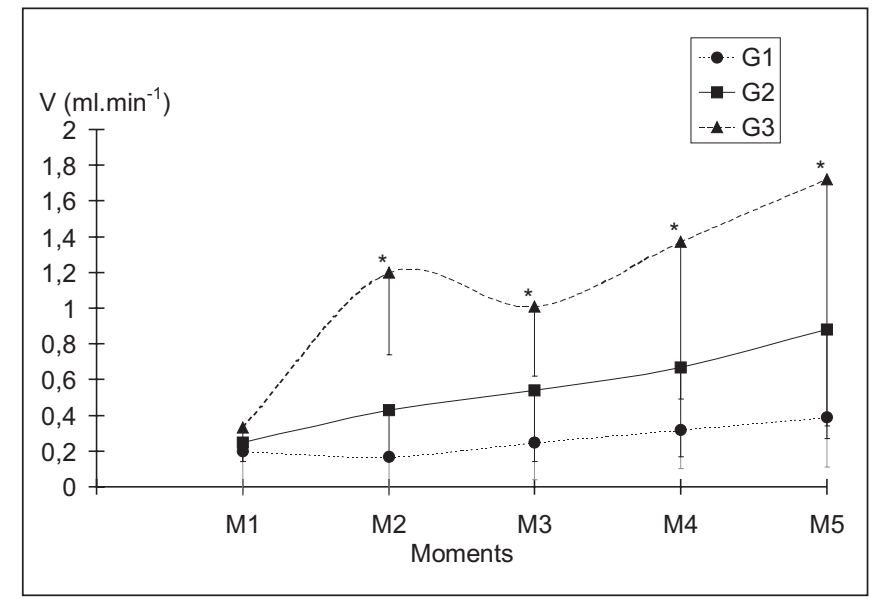

Figure 4 - Urine Volume $\left(\mathrm{ml}^{\mathrm{min}} \mathrm{m}^{-1}\right)$. Mean and Standard Deviation in each Moment for All Groups

Comment: After hemorrhage (30\% of volume replacement) and reperfusion (M1), urine volume was statistically relevan $(p<0.05)$ in $\mathrm{G3}^{*}$, with higher values as compared to other groups throughout the experiment

Knowing that hyperosmolarity is present when there is an increased number of osmotically active particles, both uremia and hypernatremia could lead to such state. However, since urea is distributed throughout body water, only an increase in plasma sodium would be able to cause hypertonicity, that is, osmotic redistribution of intracellular water to the extracellular space ${ }^{22}$.

Solutions osmotic pressure as compared to plasma is in general called tonicity. So, a fluid with osmotic pressure similar to plasma pressure is called an isotonic fluid. Hypotonic solutions have low osmotic pressure, and hypertonic solutions, such as HS and HSD, have high osmotic pressure. Osmotic pressure is approximately equal to osmolality times $19.3^{22}$ meaning that each $\mathrm{mOsm}$ generates an osmotic pressure of $19.3 \mathrm{mmHg}$ at $37^{\circ} \mathrm{C}$ through an ideal semi-patent membrane ${ }^{3}$. The solutions used in this experiment had an osmolarity of approximately $2,400 \mathrm{mOsm} . \mathrm{l}^{-1}$, thus being able to generate osmotic pressures around 8 times higher than a solution with osmolarity similar to plasma. In general, such solutions induce mild and transient hyperosmolarity ${ }^{18}$ when used in the recommended bolus doses. Upper osmolarity and plasma sodium concentration accepted levels are still to be defined ${ }^{23}$, but problems with osmolarities below $350 \mathrm{mOms} . \mathrm{I}^{-1}$ are rare and this value has not been reached by most experiments ${ }^{6,24}$. Reports on high osmolarity values are in general related to high doses or repeated injections ${ }^{25}$.

An important increase in osmolar and sodium clearance was observed in groups 2 and 3 , meaning more osmotically active substances loss through urine and natriuresis, probably caused by aldosterone secretion inhibition. This HS and HSD-induced inhibition might occur due to the fast hypovolemia correction and the installation of mild plasma hyperosmolarity. Richards et al. ${ }^{26}$ have suggested that atrial natriuretic hormone release would be the cause for this magnitude in diure- 
sis increase. However, no increase in atrial natriuretic peptide serum levels was observed in response to HSD injection 27,28

This disproportional diuresis, higher than the volume injected after HS and HSD, is observed even in the presence of hypovolemia and high antidiuretic hormone circulating concentrations. High urine production may even be noxious due to fluid loss and may be responsible for transient hypertonic solution hemodynamic effects ${ }^{29}$.

For Rocha e Silva ${ }^{18}$, hypertonic solutions interfere with endocrine secretions in animals with post-hemorrhage hypotension because they rapidly correct hypovolemia which may determine decreased vasopressin, renin and angiotensin circulating concentrations ${ }^{30}$. Decreased circulating vasopressin is seen, according to the authors, in spite of the increased osmolarity which, in general, is responsible for its secretion. In this circumstance, the response to osmolarity is overridden by hypovolemia correction, which is a vasopressin-secretion factor ${ }^{18}$.

So, hypertonic solutions determine a significant diuresis increase, which is maintained for hours after the initial injection ${ }^{15}$.

There were similarities among the three groups in free water clearance and urine osmolarity and this shows that groups receiving $\mathrm{HS}$ and $\mathrm{HSD}$ did not differ from the control group, although with higher diuresis. So, urine osmolarity was significantly decreased with time, but this was not determined by HS or HSD because the same was seen in all groups. There has been no significant differences in free water clearance, representing the excretion of solute-free water, in any moment or group.

Osmolar clearance corresponds to plasma volume released from all osmotically active substances in the time unit, that is, it is equivalent to ion clearance. As a consequence, a significant increase in ion clearance was observed in G3 due to HSD administration. This was identified by the increase in osmolar clearance curve, which initially occurred in M2, moment immediately after HSD injection. Next, there has been a mild decrease but even then it was maintained in a significantly higher level as compared to other groups. On the other hand, in G2, HS injection has determined an increased ion clearance, which was not significant but of biological importance.

The increase seen in groups 2 and 3 , which received hypertonic sodium, was possibly due to the interference of such solutions in vasopressin and aldosterone secretion. The decrease in aldosterone blood levels led to a higher osmotically active substance and sodium clearance; and vasopressin decrease has determined more urinary solute-free water loss in those groups.

HS and HSD, which have high sodium content, were responsible for plasma concentration increase of such ion. This concentration is not changed when expansion is obtained with isotonic solutions, due to renal mechanisms which would lead to a higher sodium excretion and lower reabsorbtion in all nephron segments, provided they are healthy. However, two hypertonic solutions exerting higher osmotic pressure than plasma and with a high sodium plasma concentration that goes beyond this rapid excretion ability were administered, determining an increase in this ion plasma concentration. In our experiment, this was observed in $M 2$, which is the moment immediately after hypertonic resuscitation, that is with HS and HSD infusion and, as a consequence, the most susceptible moment for significant sodium plasma concentration increases. With a $4 \mathrm{ml}^{\mathrm{kg}}{ }^{-1}$ bolus dose, hypernatremia is mild and transient ${ }^{31}$.

Similarly to sodium clearance, G3 had significant higher mean values in the urine excretion curve, showing a better ability of HSD in increasing sodium serum levels and clearance.

According to Vincent ${ }^{32}$, hypokalemia is frequently observed and probably determined by the increase in urine potassium loss. This may even cause dysrhythmias, especially in patients under digitalis. Decreased potassium serum levels were also observed by other authors ${ }^{2,16,33,34}$. The reasons for such decrease were the absence of potassium in such solutions and the fast intracellular fluid expansion, which decreases extracellular potassium.

In our study, the increase in potassium excretion with time was clearly observed.

Even following every adequate step to identify any renal change visible at electronic microscopy, no lesion compatible with post-ischemia acute tubular necrosis was observed, differently from previous reports by several authors ${ }^{35,36}$. The presence of post-ischemia acute tubular necrosis could have been seen by optic microscopy at least in G1, if the period between the end of the experiment and the removal of the organ for hystopathological study would have been longer because then one could have had enough time for necrosis delimitation.

After statistical analysis of results, it was possible to conclude that $7.5 \%$ hypertonic sodium chloride in $6 \%$ dextran 70 administered soon after mild hemorrhage and 15 minutes before a 30-minute ischemia challenge was more efficient than $7.5 \%$ hypertonic sodium chloride in protecting kidneys against ischemia-reperfussion consequences in dogs.

\section{REFERÊNCIAS - REFERENCES}

01. Brown JM, Grosso MA, Moore EE - Hypertonic saline and dextran: impact on cardiac function in the isolated rat heart. J Trauma, 1990;30:646-651.

02. Stanford GG, Patterson CR, Payne L et al - Hypertonic saline resuscitation in a porcine model of severe hemorrhagic shock. Arch Surg, 1989;124:733-736.

03. Kramer GC, Elgjo GI, De Figueiredo LFP et al Hypertonic-hyperoncotic solutions. Baillière's Clin Anaesth, 1997;II:143-161.

04. Módolo NSP, Castiglia YMM, Ganem EM et al - Acute renal ischemia model in dogs: effects of metoprolol. Ren Fail, 2001;23:1-10.

05. Szold A, Pizov R, Segal E et al - The effect of tidal volume and intravascular volume state on systolic pressure variation in ventilated dogs. Intensive Care Med, 1989;15:368-371.

06. Velasco IT, Pontieri V, Rocha e Silva M et al - Hyperosmotic $\mathrm{NaCl}$ and severe hemorrhagic shock. Am J Physiol, 1980;239: h664-h673. 
07. Wolf MB. - Plasma volume dynamics after hypertonic fluid infusing in nephrectomized dog. Am J Physiol, 1971;221:1392-1395.

08. Halvorsen L, Günther RA, Dubick MA et al - Dose response of hypertonic saline dextran solutions. J Trauma, 1991;31: 785-794.

09. Traverso LW, Bellamy RF, Hollenbach SJ - Hypertonic sodium cloride solutions: effect on hemodynamics and survival after hemorrhage in swine. J Trauma, 1987;27:32-39.

10. Nguyen TT Zwischenberger JB, Watson WC et al - Hypertonic acetate dextran achieves high-flow-low-pressure resuscitation of hemorragic shock. J Trauma, 1995;38:602-608.

11. Velasco IT, Rocha e Silva M, Oliveira MA et al - Hypertonic and hyperoncotic resuscitation from severe hemorragic shock in dogs: a comparative study. Crit Care Med, 1989;17:261-264.

12. Summary JT, Dubick MA, Zaucha GM et al - Acute and subacute toxicity of $7.5 \%$ hypertonic saline/6\% dextran (HSD) in dogs: serum immunoglobulin and complement responses. J Appl Toxicol, 1992;12:261-266.

13. Dubick MA, Wade CE - A review of the efficacy and safety of $7.5 \% \mathrm{NaCl} / 6 \%$ dextran $70 \%$ in experimental animals and in humans. J Trauma, 1994;36:323-330.

14. Mazzoni M, Warnke KC, Arfors Ket al - Capillary hemodynamics hemorrhagic shock and reperfusion: in vivo and model analysis. Am J Physiol, 1994;267:h1928-h1935.

15. Mazzoni M, Borgstrom P, Intaglietta M et al - Capillary narrowing in hemorragic shock is rectified by hyperosmotic saline-dextran reinfusion. Circ Shock, 1990;31:407-418.

16. Behrman SW, Fabian TC, Kudsk KA - Microcirculatory flow changes after initial resuscitation of hemorrhagic shock with $7.5 \%$ hypertonic saline $-6 \%$ dextran 70 . J Trauma, 1991;31: 589-560.

17. Soonden JL, Gunther RA, Dubick MA - Comparison of $7.5 \%$ $\mathrm{NaCl} / 6 \%$ dextran 70 resuscitation of hemorrhage between euhydrated sheep. Shock, 1995;3:63-68.

18. Rocha e Silva M - Hypertonic saline resuscitation: a new concept. Baillière's Clin Anaesth, 1997;Il:127-142.

19. Sztark F, Gékiere JP, Dabadie A - Effects hémodynamiques des solution salées hypertoniques. Ann Fr Anesth Réanim, 1997;16:282-291.

20. Vlahakes GJ Giamber SR, Rothaus KO - Hyperosmotic mannitol and collateral blood flow to ischemic to ischemic myocardium. J Surg Res, 1989;47:438-446.

21. Kreimeier U, Bruckner UB, Niemczyk S et al - Hyperosmotic saline dextran for resuscitation from traumatic-hemorragic hypotension: effect on regional blood flow. Circ Shock, 1990;32:83-99.

22. Prough, DS - Controverses in perioperative fluid management [IARS - Review Course Lectures]. Anesth Analg, 1994;(Suppl): 16-24.

23. Sutin KM, Ruskin KJ, Kaufman BS - Intravenous fluid therapy on neurological injury. Crit Care Clin, 1992;8:367-408.

24. Matthew CB - Treatment of hyperthermia and dehydration with hypertonic saline in dextran. Shock, 1994;2:216-221.

25. Huang PP, Stucky FS, Dimicky AR et al - Hypertonic sodium resuscitation is associated with renal failure and death. Ann Surg, 1995;221:543-557.

26. Richards AM, Nichols MG, Ikram H et al - Renal haemodynamic and hormonal effects of human alpha atrial natriuretic peptide in healthy volunteers. Lancet, 1985;545-549.

27. English TP, Weber CJ, Holcroft JW - The role of ANF in the diureses following hypertonic resuscitation. Circ Shock, $1989 ; 27: 346$.

28. Albrecht MD, Schroth M, Fahnle M et al - Effects of hypertonichyperoncotic infusion on the human atrial natriuretic factor in a standardized trial. Shock, 1995;3:152-156.
29. Cox AT, Ho HS, Gunther RA - High level of arginine vasopressin and $7.5 \% \mathrm{NaCl} / 6 \%$ dextran-70 solution: cardiovascular and renal effects. Shock, 1994;1:372-376.

30. Wade CE, Hannon JP, Bossone CA et al - Neuroendocrine responses to hypertonic saline/dextran resuscitation following hemorrhage. Circ Shock, 1991;35:37-43.

31. Shackford SR, Norton CH, Todd MM - Renal, cerebral, and pulmonary effects of hypertonic resuscitation in a porcine model of hemorrhagic shock. Surgery, 1988;104:553-560.

32. Vincent JL - Fluids for resuscitation. Br J Anaesth, 1991;67: 185-193.

33. Wade CE, Tillman FJ, Loveday JA et al - Effect of dehydratation on cardiovascular response and eletrolytes after hypertonic saline/dextran treatment for moderate hemorrhage. Ann Emerg Med, 1992;21:113-119.

34. Moon PF, Kramer GC - Hypertonic saline-dextran resuscitation from hemorrhage shock induces transient mixed acidosis. Crit Care Med, 1995;23:323-331.

35. Finn WF - Prevention of ischemic injury in renal transplantation. Kidney Int, 1990;37:171-182.

36. Honda N, Hishida A - Pathophysiology of experimental non-oliguric acute renal failure. Kidney Int, 1993;43:513-521.

\section{RESUMEN}

Rodrigues Junior GR, Amaral JLG, Castiglia YMM, Marques MEA - Eficacia de la Solución de Cloreto de Sodio a 7,5\% con y sin Dextran 70 a $6 \%$ en la Preservación de la Función Renal de Canes Hipovolémicos Sometidos a Isquemia- Reperfusión

Justificativa y Objetivos - Las soluciones hipertónicas de cloreto de sodio, asociadas o no a coloides hiperoncóticos, pueden ser eficaces en proteger el riñón en situaciones de hipovolemia. El objetivo de este estudio fue verificar en canes, el real beneficio de esas soluciones sobre la función renal, en vigencia de hipovolemia e isquemia del órgano.

Método - En 24 canes, anestesiados con pentobarbital sódico, sometidos a nefrectomia derecha y a expansión volémica con solución de Ringer. Fueron observadas posibles alteraciones renales morfo-funcionales después hemorragia de $20 \mathrm{ml}^{\mathrm{kg}} \mathrm{kg}^{-1} \mathrm{y}$ treinta minutos de total isquemia renal izquierda, con posterior reperfusión, además de la repercusión renal da administración de soluciones de cloreto de sodio 7,5\% (SH) y ésta en dextran 70 a $6 \%$ (SHD). Atributos estudiados: FC, PAM, presión de vena cava inferior, flujo sanguíneo renal, resistencia vascular renal, hematócrito, $\mathrm{Na}^{+}, \mathrm{K}^{+}$, osmolaridad plasmática, $\mathrm{PaO}_{2}$, $\mathrm{PaCO}_{2}$ y $\mathrm{pH}$, depuración (para-aminohipurato de sodio - $P A H_{-1}$, creatinina, osmolar, agua libre, $\mathrm{Na}^{+}, \mathrm{K}^{+}$), fracción de filtración, volumen y osmolaridad urinarios, excreciones urinarias $y$ fraccionarias de $\mathrm{Na}^{+}$y $\mathrm{K}^{+}$, temperatura rectal y examen histopatológico del riñón. Los atributos fueron estudiados en tres grupos (G1, G2 y G3) y en cinco momentos.

Resultados - Hubo elevación estadísticamente significativa de la presión arterial media en G2 y G3, de la resistencia vascular renal en G1, del flujo sanguíneo renal y de la depuración de $P A H$ en G3, de la excreción fraccionaria de $\mathrm{Na}^{+}$en $\mathrm{G} 2$ y G3, de las depuraciones de creatinina, osmolar, de agua libre y de $\mathrm{Na}^{+}$y $\mathrm{K}^{+}$, de la excreción urinaria de $\mathrm{Na}^{+}$y $\mathrm{K}^{+}$y del volumen urinario en $\mathrm{G} 3$. Conclusiones - La SHD administrada 15 minutos después de hemorragia moderada y 30 min antes de insulto isquémico de 30 min fue eficiente en proteger el riñón de los canes de las repercusiones de la isquemia-reperfusión. No fue constatada alteración histopatológica renal a la microscópia óptica. 